\title{
Earth-Viewing L-Band Radiometer Sensing of Sea Surface Scattered Celestial Sky Radiation-Part I: General Characteristics
}

\author{
Joseph E. Tenerelli, Nicolas Reul, Alexis A. Mouche, and Bertrand Chapron
}

\begin{abstract}
The "galactic glitter" phenomenon at L-band, i.e., the scattering of celestial sky radiation by the rough ocean surface, is examined here as a potential source of error for sea surface salinity (SSS) remote sensing. We begin by considering the transformations that must be applied to downwelling celestial noise in order to compute the eventual impact on the antenna temperature. Then, outside the context of any particular measurement system, we use approximate scattering models along with a model for the equilibrium wind wave spectrum to examine how the scattered signal at the surface might depend on the geophysical conditions and scattering geometry. It is found that, when the specular point lies far away from the galactic plane, where the incident celestial brightness is uniform, sea surface roughness has a negligible impact on the glitter. At such a point, variations in both the orientation of the incidence plane and the wind direction relative to the scattering azimuth have negligible impact. By contrast, when the specular point lies in the vicinity of a localized maximum of brightness, scattering by the roughened ocean surface may reduce the glitter by more than $30 \%$, as compared to a perfectly flat surface, and the glitter amplitude may vary by up to $0.7 \mathrm{~K}$ with variations in wind direction and by up to $0.5 \mathrm{~K}$ with variations in incidence plane orientation. It is shown that accounting for the roughness impact on celestial noise contamination is of particular concern for the remote sensing of SSS.
\end{abstract}

Index Terms-Radiometry, remote sensing, scattering.

\section{INTRODUCTION}

$\mathbf{E}$ STIMATION of the fraction of downwelling celestial sky radiation at L-band $(\simeq 1.4 \mathrm{GHz})$ that is scattered by the sea surface toward Earth-viewing radiometers is of particular concern for the remote sensing of sea surface salinity (SSS) [1]-[3]. At L-band, celestial sky radiation originates from the uniform cosmic microwave background (CMB, about $2.7 \mathrm{~K}$ ), the line emission from hydrogen, and a continuum background [3], [4]. The intensity of scattered celestial radiation depends on the source intensity, the surface roughness, the observation geometry, and the antenna characteristics (e.g., the gain pattern).

Manuscript received March 1, 2007; revised August 1, 2007.

J. E. Tenerelli, N. Reul, and B. Chapron are with the Laboratoire d'Océanographie Spatiale, Institut Français de Recherche et d'Exploitation de la Mer, 29200 Plouzané, France (e-mail: joseph.tenerelli@ifremer.fr).

A. A. Mouche is with the Laboratoire d'Océanographie Spatiale, Institut Français de Recherche et d'Exploitation de la Mer, 29200 Plouzané, France, and also with the Centre National d'Étude Spatiales, 75039 Paris, France.

Color versions of one or more of the figures in this paper are available online at http://ieeexplore.ieee.org.

Digital Object Identifier 10.1109/TGRS.2007.914803
For a perfectly flat surface with a reflectivity of unity, Le Vine and Abraham [3] reported that the total effective impact of celestial noise on antenna temperature (i.e., the integral over the antenna gain pattern of the appropriately normalized line emission, continuum, and CMB) for a sun-synchronous orbiting instrument with a full-width at half-maximum on the order of $10^{\circ}$, an orbital inclination of $95^{\circ}$, and a 6 A.M./ 6 P.M. equatorial crossing time ranges from about $4 \mathrm{~K}$ to more than $9 \mathrm{~K}$. This impact varies with the orientation of the sensor, the spacecraft location along the orbit, and the time of year. Relative to this variation, the expected dynamical range of sea surface brightness temperature change at L-band due to SSS variation is small and typically does not exceed $4 \mathrm{~K}$ for open ocean conditions [2]. Therefore, the systematic and significant variability of sea surface reflected celestial radiation might hamper accurate SSS retrievals from spaceborne measurements of upwelling L-band brightness temperatures.

The line emission from hydrogen and the continuum background are spatially inhomogeneous and strongest in the direction of the equatorial plane of the galaxy and at several localized strong spots (e.g., Cassiopeia A and Cygnus A). Therefore, forthcoming missions dedicated to SSS remote sensing on sunsynchronous satellite platforms (e.g., European Space Agency Soil Moisture and Ocean Salinity (SMOS) mission and the National Aeronautics and Space Administration/Comisión Nacional de Actividades Espaciales Aquarius/SAC-D mission) are expected to be geographically and seasonally affected.

Le Vine and Abraham [3] introduced a method to produce an unpolarized map of the equivalent brightness temperature of radiation at L-band from the $\mathrm{CMB}$, from the hydrogen line $T_{\mathrm{HI}}$, and from the continuum background $T_{\text {cont }}$, based on recent radio astronomy surveys [5]-[10]. It was shown in [4] that the model given in [3] is generally consistent with measurements made with several modern remote sensing instruments directly pointing toward the sky, although the data suggest a slight polarization signature. To correct for reflected celestial noise in radiometric L-band data acquired during scientific campaigns, investigators have assumed that the ocean surface is perfectly flat [11]-[14]. The same assumption was used in [2] and [15] to provide preliminary estimates of the expected reflected celestial radiation for the future Aquarius/SAC-D and SMOS missions, respectively. Over a flat sea surface, the L-band reflectivity varies from about $50 \%$ to $80 \%$ for incidence angles below $60^{\circ}$. Considering the available radiometric data collected at L-band in this incidence angle range over water surfaces [12]-[14], 
[16]-[20], the sensitivity of surface horizontally and vertically polarized emissivity to surface roughening by the wind is never observed to exceed $2 \times 10^{-3} /(\mathrm{m} / \mathrm{s})$. The maximum sensitivity of the reflectivity will be the same, so that, for an increase in wind speed of $10 \mathrm{~m} / \mathrm{s}$, the horizontally and vertically polarized reflectivities will decrease from the corresponding flat surface values by less than $2 \%$. Therefore, if celestial noise were spatially uniform, the impact of sea surface roughness on the celestial contamination would be negligible in the context of SSS retrievals. However, celestial noise is not spatially uniform, and this nonuniformity may have a significant impact on the wind speed dependence of the scattered noise. In the analysis of radiometer data obtained during the L-band Ocean Salinity Airborne Campaign [11], it was found that the impact of celestial noise on the linear channel antenna temperatures ranged from $90 \%$ to $60 \%$ of that computed using the Fresnel power reflection coefficients for wind speeds ranging from 0 to $20 \mathrm{~m} / \mathrm{s}$, respectively.

This effect is a consequence of the angular spreading of the reflected noise and, to a lesser extent, the reduction of the reflectivity in the presence of roughness. In this paper, we analyze forward scattering of L-band celestial radiation by the rough ocean surface using approximate scattering models together with a spectral model for wind waves in statistical equilibrium. In the first part of the paper (hereafter referred to as Part I), we consider the general characteristics of the rough sea surface scattered L-band celestial noise. In Part II [52], we analyze the impact of scattered celestial noise on antenna temperature for the specific case of SMOS, with its characteristic multiangular sensing, and we characterize the expected annual cycle of the contamination for that mission.

In order to place the scattering calculations in context and to reveal key assumptions made in the development, in Section II, we review the transformations that the celestial sky radiation undergoes as it propagates from the source to the surface where it is scattered toward an Earth-viewing radiometer. In Section III, we review the rough surface and electromagnetic scattering models employed in the calculations. In Section IV, we consider the geometry of the problem and develop an efficient functional representation of the scattered signal. Next, in Section $\mathrm{V}$, we examine the extent to which the rough sea surface spreads the scattered celestial noise away from the specular direction. As our main concern is to analyze the effect of surface roughness on angular spreading of the celestial noise, we neglect atmospheric attenuation on the downward path to the surface, and we assume that the incoming radiation is unpolarized, so that we may ignore downward Faraday rotation. Then, having established the basic tendency of the roughened sea surface to spread the scattered celestial noise over the specular lobe, in Section VI, we consider in more detail how this spreading effect depends on radiometer incidence angle, wind direction, and the orientation of the incidence plane in the celestial frame. We examine the solutions at a "cold" specular point far away from the galactic equator and at a "hot" specular point near the galactic plane, and we find significantly different behavior at these two points. Finally, in Section VII, we discuss the remaining issues and sources of uncertainty in the solutions.

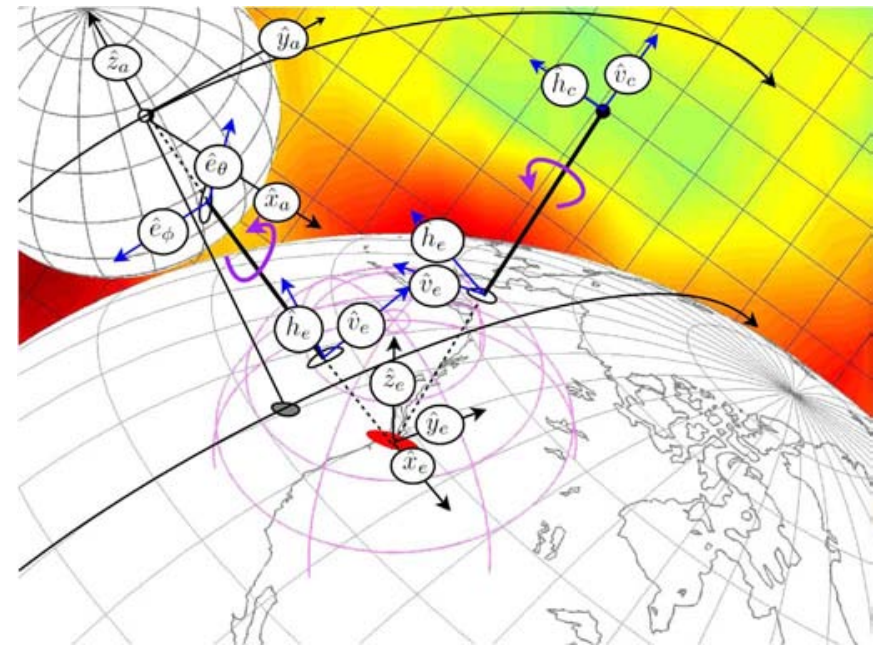

Fig. 1. Diagram showing the reference frames involved in the surface scattering problem along with celestial noise in the background. The sphere in the upper left corner represents the spherical coordinate system in the instrument (or antenna) frame, whose associated Cartesian basis vectors are $\left(\hat{\mathbf{x}}_{\mathbf{a}}, \hat{\mathbf{y}}_{\mathbf{a}}, \hat{\mathbf{z}}_{\mathbf{a}}\right)$. The topocentric, or target, frame centered at the surface target (indicated by the red oval on Earth) has Cartesian basis vectors denoted by $\left(\hat{\mathbf{x}}_{\mathbf{e}}, \hat{\mathbf{y}}_{\mathbf{e}}, \hat{\mathbf{z}}_{\mathbf{e}}\right)$, and the associated spherical coordinate system is represented by the light magenta azimuth and elevation contours. Polarization basis vectors for the celestial, target, and instrument frames are indicated by blue arrows. Horizontal and vertical polarization basis vectors in the celestial frame are denoted by $\hat{\mathbf{h}}_{\mathbf{c}}$ and $\hat{\mathbf{v}}_{\mathbf{c}}$, respectively. Horizontal and vertical polarization basis vectors for both incident and scattered fields at the target are denoted by $\hat{\mathbf{h}}_{\mathbf{e}}$ and $\hat{\mathbf{v}}_{\mathbf{e}}$, respectively, and these are defined using the FSA convention [21]. Horizontal and vertical polarization basis vectors in the instrument (or antenna) frame are denoted by $\hat{\mathbf{e}}_{\phi}$ and $\hat{\mathbf{e}}_{\theta}$, respectively. Long curved arrows show the path of the satellite. Heavy magenta arrows show clockwise (looking in the radiation propagation direction) polarization basis rotations.

\section{TRACing the Celestial RADiation FROM SOURCE TO RECEIVER}

In evaluating the impact of celestial sky radiation on polarimetric measurements, the quantities of primary interest are the elements of the modified Stokes vector

$$
\left(\begin{array}{c}
T_{h}\left(\theta_{\circ}, \phi_{\circ}, t\right) \\
T_{v}\left(\theta_{\circ}, \phi_{\circ}, t\right) \\
U\left(\theta_{\circ}, \phi_{\circ}, t\right) \\
V\left(\theta_{\circ}, \phi_{\circ}, t\right)
\end{array}\right)=\mathcal{K}\left(\begin{array}{c}
\left\langle E_{h}\left(\theta_{\circ}, \phi_{\circ}, t\right) E_{h}^{*}\left(\theta_{\circ}, \phi_{\circ}, t\right)\right\rangle \\
\left\langle E_{v}\left(\theta_{\circ}, \phi_{\circ}, t\right) E_{v}^{*}\left(\theta_{\circ}, \phi_{\circ}, t\right)\right\rangle \\
2 \Re\left\langle E_{v}\left(\theta_{\circ}, \phi_{\circ}, t\right) E_{h}^{*}\left(\theta_{\circ}, \phi_{\circ}, t\right)\right\rangle \\
2 \Im\left\langle E_{v}\left(\theta_{\circ}, \phi_{\circ}, t\right) E_{h}^{*}\left(\theta_{\circ}, \phi_{\circ}, t\right)\right\rangle
\end{array}\right)
$$

where $E_{p}$ are orthogonal electrical field components at polarization $p, t$ is time, $\left(\theta_{\circ}, \phi_{\circ}\right)$ specifies the propagation direction, and \langle\rangle denotes an ensemble average. The constant $\mathcal{K}=\lambda^{2} /\left(k_{b} \eta\right)$, where $\lambda$ is the radiation wavelength, $k_{b}$ is Boltzmann's constant, and $\eta$ is the intrinsic impedance of free space. Here, we use the notation $\mathbf{T}_{\mathbf{p}}$ to denote the modified Stokes vector and $T_{p}$ to denote a particular component of the Stokes vector.

In what follows, we describe the transformation from source to receiver in the general case of polarized celestial sky radiation but also treat the simpler case of an unpolarized source. As shown in Fig. 1, when celestial radiation propagates from the source to an Earth-viewing receiver with intermediate scattering at the ocean surface, it undergoes several transformations, some of which simply involve a change in polarization basis. 


\section{A. From the Source to the Ocean Surface}

The first transformation that must be applied to polarized radiation emitted by a celestial source involves the change of electric field basis from the celestial basis, with electric field $\left(E_{h c}, E_{v c}\right)^{T}$ and associated Stokes vector $\left(T_{h c}, T_{v c}, U_{c}, V_{c}\right)^{T}$, to the ocean surface target basis, with electric field $\left(E_{h e}, E_{v e}\right)^{T}$ and associated Stokes vector $\left(T_{h e}, T_{v e}, U_{e}, V_{e}\right)^{T}$. Celestial polarization basis vectors $\hat{\mathbf{h}}_{\mathbf{c}}$ and $\hat{\mathbf{v}}_{\mathbf{c}}$ may be defined in terms of the vector from the Earth's center pointing toward the celestial North Pole $\hat{\mathbf{n}}_{\mathbf{c}}$ and the energy propagation direction $\hat{\mathbf{k}}$. The polarization basis vectors for the radiation incident at the target may be defined in terms of the local normal to the horizontal surface $\hat{\mathbf{z}}_{\mathbf{e}}$ and the energy propagation direction $\hat{\mathbf{k}}$. Here, we use the forward scattering alignment (FSA) convention [21], in which $\hat{\mathbf{v}} \times \hat{\mathbf{h}}$ is in the radiation propagation direction for both the incident and scattered fields. Following [22], the transformation between the celestial and target incident polarization basis vectors may be expressed as a counterclockwise rotation of orthogonal electric field components $\left(E_{h}, E_{v}\right)^{T}$ about the propagation direction by angle $\Psi$, and the corresponding Stokes vector transformation is

$$
\left(\begin{array}{c}
T_{h e} \\
T_{v e} \\
U_{e} \\
V_{e}
\end{array}\right)=\mathbf{M}_{\Psi}\left(\begin{array}{c}
T_{h c} \\
T_{v c} \\
U_{c} \\
V_{c}
\end{array}\right)
$$

where

$$
\mathbf{M}_{\Psi}=\left(\begin{array}{cccc}
\cos ^{2} \Psi & \sin ^{2} \Psi & -\cos \Psi \sin \Psi & 0 \\
\sin ^{2} \Psi & \cos ^{2} \Psi & \cos \Psi \sin \Psi & 0 \\
\sin (2 \Psi) & -\sin (2 \Psi) & \cos (2 \Psi) & 0 \\
0 & 0 & 0 & 1
\end{array}\right)
$$

Next, accounting for Faraday rotation across the ionosphere [23] and attenuation across the atmosphere [24] on the downward path, the downwelling sky radiation Stokes vector becomes

$$
\left(\begin{array}{c}
T_{h e}^{\prime} \\
T_{v e}^{\prime} \\
U_{e}^{\prime} \\
V_{e}^{\prime}
\end{array}\right)=\mathbf{A}_{\mathbf{d}}\left(\tau\left(\theta_{\circ}\right)\right) \mathbf{M}_{\mathbf{f}}\left(\omega_{f d}\right)\left(\begin{array}{c}
T_{h e} \\
T_{v e} \\
U_{e} \\
V_{e}
\end{array}\right)
$$

where $\mathbf{M}_{\mathbf{f}}\left(\omega_{f d}\right)$ and $\mathbf{A}_{\mathbf{d}}\left(\tau\left(\theta_{\circ}\right)\right)$ are Faraday rotation and downward atmospheric attenuation matrices, respectively. The former is

$\mathbf{M}_{\mathbf{f}}\left(\omega_{f d}\right)=\left(\begin{array}{cccc}\cos ^{2} \omega_{f d} & \sin ^{2} \omega_{f d} & -\cos \omega_{f d} \sin \omega_{f d} & 0 \\ \sin ^{2} \omega_{f d} & \cos ^{2} \omega_{f d} & \cos \omega_{f d} \sin \omega_{f d} & 0 \\ \sin \left(2 \omega_{f d}\right) & -\sin \left(2 \omega_{f d}\right) & \cos \left(2 \omega_{f d}\right) & 0 \\ 0 & 0 & 0 & 1\end{array}\right)$

which corresponds to a counterclockwise rotation of $\left(E_{h e}, E_{v e}\right)^{T}$ by an angle $\omega_{f d}$, which is a function of the total electron content as well as the Earth's magnetic field. Downward atmospheric attenuation matrix $\mathbf{A}_{\mathbf{d}}\left(\tau\left(\theta_{\circ}\right)\right)$ is given by

$$
\mathbf{A}_{\mathbf{d}}\left(\tau\left(\theta_{\circ}\right)\right)=\left(\begin{array}{cccc}
\tau\left(\theta_{\circ}\right) & 0 & 0 & 0 \\
0 & \tau\left(\theta_{\circ}\right) & 0 & 0 \\
0 & 0 & \tau\left(\theta_{\circ}\right) & 0 \\
0 & 0 & 0 & \tau\left(\theta_{\circ}\right)
\end{array}\right)
$$

where $\tau\left(\theta_{\circ}\right)=e^{-a \sec \theta_{\circ}}$ is the one-way slantwise power transmittance through the atmosphere at angle $\theta_{\circ}$ from zenith. At L-band, the one-way zenith power attenuation $a$ (in nepers) depends mostly on the oxygen and (to a much lesser extent) water vapor concentrations of the atmosphere [24].

\section{B. Sea Surface Scattering}

Next, the radiation is scattered by the rough ocean surface. As discussed further in Section III, rough surface scattering models provide expressions for the scattering amplitudes, from which normalized scattering cross sections may be analytically determined in terms of the statistics of the rough sea surface. The Stokes vector of the scattered field $\left(T_{h e}^{g s}, T_{v e}^{g s}, U_{e}^{g s}, V_{e}^{g s}\right)^{T}$ associated with rough surface scattering of the incident field with Stokes vector $\left(T_{h e}, T_{v e}, U_{e}, V_{e}\right)^{T}$ is

$$
\left(\begin{array}{c}
T_{h e}^{g s} \\
T_{v e}^{g s} \\
U_{e}^{g s} \\
V_{e}^{g s}
\end{array}\right)=\mathbf{M}_{\mathbf{s}}\left(\begin{array}{c}
T_{h e}^{\prime} \\
T_{v e}^{\prime} \\
U_{e}^{\prime} \\
V_{e}^{\prime}
\end{array}\right)
$$

where $\mathbf{M}_{\mathbf{s}}$ is the Mueller matrix [21], [25]. This equation is an expression for the scattered brightness temperatures in some direction $\left(\theta_{s}, \phi_{s}\right)$ associated with radiation incident at the target from a particular direction $\left(\theta_{\circ}, \phi_{\circ}\right)$ in the upper hemisphere. The total scattered field Stokes vector in a given direction $\left(\theta_{s}, \phi_{s}\right)$ is obtained by integrating the contributions from all directions in the upper hemisphere, i.e.,

$$
\tilde{\mathbf{T}}_{\mathbf{p}}^{\mathbf{g s}}\left(\theta_{s}, \phi_{s}\right)=\frac{1}{4 \pi \cos \theta_{s}} \int_{\Omega_{\circ}}\left(\mathbf{M}_{\mathbf{s}} \mathbf{A}_{\mathbf{d}} \mathbf{M}_{\mathbf{f d}} \mathbf{M}_{\mathbf{\Psi}}\right) \mathbf{T}_{\mathbf{p}} d \Omega_{\circ}
$$

where $\mathbf{M}_{\mathbf{f d}}=\mathbf{M}_{\mathbf{f}}\left(\omega_{\mathbf{f d}}\right)$, and $\Omega_{\circ}$. refers to the entire upper hemisphere solid angle $\left(d \Omega_{\circ}=\sin \theta_{\circ} d \phi_{\circ} d \theta_{\circ}\right)$.

When considering the simple case of (assumed) unpolarized celestial sky radiation with brightness temperature $\mathbf{T}_{\mathbf{p}}\left(\Omega_{0}\right)=$ $T_{\text {sky }}\left(\Omega_{\circ}\right)$, both $\mathbf{M}_{\mathbf{f d}}$ and $\mathbf{M}_{\boldsymbol{\Psi}}$ may be replaced by the $4 \times 4$ identity matrix I. If, in addition to this approximation, one assumes a simple exponential model for atmospheric attenuation [24], then the brightness temperature for component $p$ (here, we consider only horizontal and vertical linear polarizations) of the scattered celestial noise Stokes vector reduces to

$$
\begin{aligned}
\tilde{\mathbf{T}}_{\mathbf{p}}^{\mathbf{g s}}\left(\theta_{s}, \phi_{s}\right)= & \frac{1}{4 \pi \cos \theta_{s}} \int_{\Omega_{\circ}}\left[\sigma_{p p}\left(\theta_{s}, \phi_{s}, \Omega_{\circ}\right)+\sigma_{p q}\left(\theta_{s}, \phi_{s}, \Omega_{\circ}\right)\right] \\
& \times T_{\text {sky }}\left(\Omega_{\circ}\right) e^{-a \sec \theta_{\circ}\left(\Omega_{\circ}\right)} d \Omega_{\circ}
\end{aligned}
$$

where $\sigma_{p q}\left(\theta_{s}, \phi_{s}, \Omega_{\circ}\right)$ are the normalized (dimensionless) bistatic scattering cross sections of the sea surface, which are 
functions of both the scattered wave direction $\left(\theta_{s}, \phi_{s}\right)$ and the incident wave direction $\left(\theta_{0}, \phi_{\circ}\right)$. The dependence of the cross sections on the rough surface state parameters, target location on Earth, and time is implicit.

\section{From the Surface to the Radiometer}

After surface scattering, the signal is slightly attenuated by the atmosphere (with one-way slantwise power transmittance $\left.\tau\left(\theta_{s}\right)=e^{-a \sec \theta_{s}}\right)$ on the upward path toward the radiometer, i.e.,

$$
\begin{aligned}
\left(\begin{array}{c}
T_{h e}^{g s^{\prime}} \\
T_{v e}^{g s^{\prime}} \\
U_{e}^{g s^{\prime}} \\
V_{e}^{g s^{\prime}}
\end{array}\right) & =\left(\begin{array}{cccc}
\tau\left(\theta_{s}\right) & 0 & 0 & 0 \\
0 & \tau\left(\theta_{s}\right) & 0 & 0 \\
0 & 0 & \tau\left(\theta_{s}\right) & 0 \\
0 & 0 & 0 & \tau\left(\theta_{s}\right)
\end{array}\right)\left(\begin{array}{c}
T_{h e}^{g s} \\
T_{v e}^{g s} \\
U_{e}^{g s} \\
V_{e}^{g s}
\end{array}\right) \\
& =\mathbf{A}_{\mathbf{u}}\left(\theta_{s}\right)\left(\begin{array}{c}
T_{h e}^{g s} \\
T_{v e}^{g s} \\
U_{e}^{g s} \\
V_{e}^{g s}
\end{array}\right)
\end{aligned}
$$

and, possibly, affected Faraday rotation across the ionosphere. Faraday rotation leads to a counterclockwise rotation of $\left(E_{h e}^{g s^{\prime}}, E_{v e}^{g s^{\prime}}\right)^{T}$ by the angle $\omega_{f u}$, and the corresponding Stokes vector transformation is

$$
\left(\begin{array}{c}
T_{h e}^{g s^{\prime \prime}} \\
T_{v e}^{g s^{\prime \prime}} \\
U_{e}^{g s^{\prime \prime}} \\
V_{e}^{g s^{\prime \prime}}
\end{array}\right)=\mathbf{M}_{\mathbf{f}}\left(\omega_{f u}\right)\left(\begin{array}{c}
T_{h e}^{g s^{\prime}} \\
T_{v e}^{g s^{\prime}} \\
U_{e}^{g s^{\prime}} \\
V_{e}^{g s^{\prime}}
\end{array}\right)
$$

where Faraday rotation matrix $\mathbf{M}_{\mathbf{f}}$ was previously defined. For notational simplicity, we let $\mathbf{M}_{\mathbf{f}}\left(\omega_{f u}\right)=\mathbf{M}_{\mathbf{f u}}$ in the following. Next, the electric field components are transformed from the target basis $\left(\hat{\mathbf{h}}_{\mathbf{e}}, \hat{\mathbf{v}}_{\mathbf{e}}\right)$ to a suitable basis in the antenna frame $\left(\hat{\mathbf{e}}_{\theta}, \hat{\mathbf{e}}_{\phi}\right)$. This involves a clockwise (looking toward the antenna from the target) rotation of the basis vectors about the line of sight by the Claassen polarization basis rotation angle $\Psi_{l}$ [22]. The resulting Stokes vector is then transformed into the Ludwig-3 antenna basis [26] by an additional rotation about the line of sight by the azimuth angle $\phi_{a}$ in the antenna frame. This angle is measured clockwise from the $\hat{\mathbf{x}}_{\mathbf{a}}$-axis, looking down the $\hat{\mathbf{z}}_{\mathrm{a}}$-axis toward the antenna. Letting $\Phi_{l}=\phi_{a}-\Psi_{l}$ be the polarization basis clockwise rotation angle associated with the combined Claassen and Ludwig-3 transformations, the Stokes vector in the Ludwig-3 basis $\left(T_{h e}^{g g^{\prime \prime \prime}}, T_{v e}^{g s^{\prime \prime \prime}}, U_{e}^{g s^{\prime \prime \prime}}, V_{e}^{g s^{\prime \prime \prime}}\right)^{T}$ is

$$
\left(\begin{array}{c}
T_{h e}^{g s^{\prime \prime \prime}} \\
T_{v e}^{g s^{\prime \prime \prime}} \\
U_{e}^{g s^{\prime \prime \prime}} \\
V_{e}^{g s^{\prime \prime \prime}}
\end{array}\right)=\mathbf{M}_{\mathbf{l}}\left(\begin{array}{c}
T_{h e}^{g s^{\prime \prime}} \\
T_{v e}^{g s^{\prime \prime}} \\
U_{e}^{g s^{\prime \prime}} \\
V_{e}^{g s^{\prime \prime}}
\end{array}\right)
$$

where polarization basis rotation matrix $\mathbf{M}_{\mathbf{l}}$ is defined by

$$
\mathbf{M}_{\mathbf{l}}=\left(\begin{array}{cccc}
\cos ^{2} \Phi_{l} & \sin ^{2} \Phi_{l} & -\cos \Phi_{l} \sin \Phi_{l} & 0 \\
\sin ^{2} \Phi_{l} & \cos ^{2} \Phi_{l} & \cos \Phi_{l} \sin \Phi_{l} & 0 \\
\sin \left(2 \Phi_{l}\right) & -\sin \left(2 \Phi_{l}\right) & \cos \left(2 \Phi_{l}\right) & 0 \\
0 & 0 & 0 & 1
\end{array}\right) .
$$

The final impact of the scattered celestial noise on antenna temperature may be computed by applying the preceding transformations to the scene brightness temperatures, applying the antenna gain pattern, and then integrating over the scene.

To recapitulate the preceding results, the brightness temperature at polarization $p$ in direction $\left(\theta_{a}, \phi_{a}\right)$ in the antenna frame associated with scattered celestial noise, after all transformations from the source to the antenna (but prior to weighting by the antenna gain), is

$$
\begin{aligned}
\tilde{\mathbf{T}}_{\mathbf{p}}^{\mathbf{a}}\left(\theta_{a}, \phi_{a}\right)= & \left(\mathbf{M}_{\alpha} \mathbf{A}_{\mathbf{u}}\right) \tilde{\mathbf{T}}_{\mathbf{p}}^{\mathbf{g s}}=\frac{1}{4 \pi \cos \theta_{s}} \\
& \times\left(\mathbf{M}_{\alpha} \mathbf{A}_{\mathbf{u}}\right) \int_{\Omega_{\circ}}\left(\mathbf{M}_{\mathbf{s}} \mathbf{A}_{\mathbf{d}} \mathbf{M}_{\mathbf{f d}} \mathbf{M}_{\Psi}\right) \mathbf{T}_{\mathbf{p}} d \Omega_{\circ}
\end{aligned}
$$

where we have followed convention (e.g., see [27]) and combined $\mathbf{M}_{\mathbf{l}} \mathbf{M}_{\mathbf{f u}}$ into one rotation matrix $\mathbf{M}_{\alpha}=\mathbf{M}_{\mathbf{l}} \mathbf{M}_{\mathbf{f u}}$. When weighted by the antenna gain, $\tilde{\mathbf{T}}_{\mathbf{p}}^{\mathbf{a}}\left(\theta_{a}, \phi_{a}\right)$ yields the contribution to the antenna temperature from the scattered noise incident at the antenna from direction $\left(\theta_{a}, \phi_{a}\right)$; to obtain the antenna temperature Stokes vector, contributions from all directions must be integrated, and the contribution to the total antenna temperature Stokes vector from scattered celestial noise is

$$
\overline{\mathbf{T}}_{\mathbf{p}}^{\mathbf{a}}=\frac{1}{\Omega_{a}} \int_{\Omega_{a}} \frac{\left(\mathbf{G} \mathbf{M}_{\alpha} \mathbf{A}_{\mathbf{u}}\right)}{4 \pi \cos \theta_{s}} \int_{\Omega_{\circ}}\left(\mathbf{M}_{\mathbf{s}} \mathbf{A}_{\mathbf{d}} \mathbf{M}_{\mathbf{f d}} \mathbf{M}_{\Psi}\right) \mathbf{T}_{\mathbf{p}} d \Omega_{\circ} d \Omega_{a}
$$

where $\Omega_{a}$ appropriately normalizes antenna gain pattern matrix $\mathbf{G}$.

Considering again the simple case of unpolarized celestial radiation with brightness temperature $T_{\mathrm{sky}}\left(\Omega_{\circ}\right)$ and assuming a simple exponential model for attenuation on both the downward and upward paths, the antenna temperature Stokes component $p$ (where $p$ refers to either horizontal or vertical linear polarization) due to rough sea scattered celestial radiation reduces to

$$
\begin{array}{r}
\overline{\mathbf{T}}_{\mathbf{p}}^{\mathbf{a}}=\frac{1}{\Omega_{a}} \int_{\Omega_{a}} \frac{\left(\mathbf{G M}_{\alpha}\right)}{4 \pi \cos \theta_{s}} e^{-a \sec \theta_{s}} \int_{\Omega_{\circ}\left(\Omega_{a}\right)}\left[\sigma_{p p}\left(\theta_{s}, \phi_{s}, \Omega_{\circ}\right)\right. \\
\left.\quad+\sigma_{p q}\left(\theta_{s}, \phi_{s}, \Omega_{\circ}\right)\right] e^{-a \sec \theta_{\circ}} T_{\text {sky }}\left(\Omega_{\circ}\right) d \Omega_{\circ} d \Omega_{a}
\end{array}
$$

As formulated, (15) presents some difficulty for practical implementation since the zenith atmospheric attenuation $a$ depends on the atmospheric state. The impact of this factor is more significant at very large incidence angles beyond $60^{\circ}$, and since we do not focus on such incidence angles, we simplify the problem and neglect atmospheric attenuation on both the downward and upward paths. A possible solution for this difficulty may involve the use of standard atmospheric profiles to evaluate the zenith atmospheric attenuation within the integration over the incident celestial noise, but further work is required to evaluate this strategy. 


\section{ROUGH SURFACE SCATTERING}

\section{A. Bistatic Scattering Models at L-Band}

A key component required for evaluating celestial glitter contributions at L-band is a formulation for the bistatic scattering cross sections of the rough sea surface. In [28], rough sea surface scattering of solar radiation at L-band was discussed in detail. As shown in [28], two approximate solutions to the rough surface scattering problem may be considered to provide asymptotic limits within a common framework. These approximate solutions are the Kirchhoff approximation (KA) [29] and the first-order small-slope approximation (SSA-1) [30].

Application of either the SSA-1 or the KA for scattering from the slightly rough ocean surface yields the following expression for the dimensionless bistatic scattering cross sections $\sigma_{\alpha \alpha_{\circ}}^{\circ}$ for the scattering of the incoming wave of polarization $\alpha_{\circ}$ into the outgoing wave of polarization $\alpha$ [31]:

$$
\begin{aligned}
\sigma_{\alpha \alpha_{\circ}}\left(\mathbf{k}_{\mathbf{s}}, \mathbf{k}_{\circ}\right)=\frac{1}{\pi} \mid \frac{2 q_{s} q_{\circ}}{q_{s}+} q_{\circ} & \left.B_{\alpha \alpha_{\circ}}\left(\mathbf{k}_{s}, \mathbf{k}_{\circ}\right)\right|^{2} \\
& \times \exp \left\{-\left(q_{s}+q_{\circ}\right)^{2} \rho(0)\right\} \cdot I_{K}
\end{aligned}
$$

where Kirchhoff integral $I_{K}$ is given in Cartesian coordinates by

$$
\begin{aligned}
I_{K}=\iint\left[\exp \left\{\left(q_{s}+q_{\circ}\right)^{2} \rho(\mathbf{x})\right\}-1\right] \\
\\
\times \exp \left\{-i\left(\mathbf{k}_{\mathbf{s}}-\mathbf{k}_{\circ}\right) \cdot \mathbf{x}\right\} d x d y
\end{aligned}
$$

and where the integration domain extends from $-\infty$ to $+\infty$ in each dimension. Vector $\mathbf{x}$ is the horizontal displacement, and the integral is evaluated over all possible displacements in the horizontal plane. In (16) and (17), $\mathbf{k}_{\mathbf{s}}$ and $\mathbf{k}_{\circ}$ are the scattered and incident wave vectors, respectively; $q_{s}=\hat{\mathbf{z}}_{\mathbf{e}} \cdot \mathbf{k}_{\mathbf{s}}$ and $q_{\circ}=-\hat{\mathbf{z}}_{\mathbf{e}} \cdot \mathbf{k}_{\circ}$ are the vertical projections of the scattered and incident wave vectors, respectively; the kernel functions $B_{\alpha \alpha_{\circ}}\left(\mathbf{k}_{\mathbf{s}}, \mathbf{k}_{\circ}\right)$ are functions of both the scattering geometry and the dielectric constant, and they take different forms in the SSA-1 and the KA. Explicit expressions for these kernel functions may be found in [31] and [32]. The dielectric constant for seawater at L-band is obtained from the Klein and Swift model [33].

In this paper, the sea surface elevation function is assumed to be a Gaussian random process, and the correlation function $\rho(\mathbf{x})$ of the surface elevation is obtained from the Fourier transform of the roughness spectrum. Here, we use the equilibrium wave spectrum model of Kudryavtsev et al. [34]. Note that the second-order moment of this spectrum, namely, the integrated mean square slope (MSS), is constrained to agree with the Cox and Munk [35] clean (and optionally slick) surface results. This spectral model primarily depends on the $10-\mathrm{m} / \mathrm{s}$ wind speed $u_{10}$, wind direction $\varphi_{w}$, and inverse wave age $\Omega$. Here, we take the inverse wave age to be 0.83 , which corresponds to a fully developed sea. A change in the wave age will mainly affect the characteristics of the larger gravity waves around the spectral peak, and this will mostly impact the scattering behavior in the vicinity of the specular direction. While the choice of sea surface spectrum model used in the calculation of the scattered celestial noise is an important issue, the chosen spectrum follows physical principles and has been shown to provide consistent comparisons with both optical and active microwave measurements [36], [37].

In [28, Appendix], it is shown that the twofold integration (17) may be reduced to a 1-D integral using an azimuthal harmonic decomposition of the integrand in polar coordinates. Incorporating the polarization-dependent coefficients multiplied with the Kirchhoff integral $I_{K}$ into a sum over the Kirchhoff integral harmonics yields

$$
\begin{aligned}
\sigma_{\alpha \alpha_{\circ}}\left(\mathbf{k}_{\mathbf{s}}, \mathbf{k}_{\circ}, u_{10}, \varphi_{w}\right)=\sum_{m=0}^{\infty} \sigma_{\alpha \alpha_{\circ}}^{(2 m)}\left(\mathbf{k}_{\mathbf{s}}, \mathbf{k}_{\circ}, u_{10}\right) \\
\quad \times \cos 2 m\left(\Phi_{s i}-\varphi_{w}\right)
\end{aligned}
$$

where $\sigma_{\alpha \alpha_{o}}^{(2 m)}$ are the azimuthal harmonics of the normalized bistatic scattering cross sections, and $\Phi_{s i}$ is the azimuth angle of $\mathbf{q}=\mathbf{k}_{\mathbf{s}}-\mathbf{k}_{\circ}$. We have explicitly included the dependence of the final scattering cross sections on wind speed $u_{10}$ and downwind direction $\varphi_{w}$.

Note that the scattering cross section harmonics $\sigma_{\alpha \alpha_{0}}^{(2 m)}$ are independent of wind direction. Moreover, these harmonics only depend on the incident and scattered radiation incidence angles, the wind speed, and the difference between the incident and scattered radiation azimuth angles. To facilitate efficient scattering calculations, we precompute the SSA- 1 and the KA $\sigma_{\alpha \alpha_{\circ}}^{(2 m)}$ functions for a set of $\left(\theta_{\circ}, \phi_{s}-\phi_{\circ}, \theta_{s}, u_{10}\right)$ values.

\section{B. Surface Salinity and Temperature Dependencies}

As previously noted, in this paper, we use the Klein and Swift model [33] to evaluate the scattered celestial noise. According to dielectric models such as Klein and Swift, for typical ocean values of sea surface temperature (SST) $\left(0{ }^{\circ} \mathrm{C}\right.$ to $\left.38{ }^{\circ} \mathrm{C}\right)$ and SSS (20-40 psu), the sensitivity of the L-band flat surface emissivity (and reflectivity) to varying SSS ranges from about $0.5 \times 10^{-3} / \mathrm{psu}$ to $3.5 \times 10^{-3} / \mathrm{psu}$, and the sensitivity to varying SST ranges from about $0.2 \times 10^{-3} /{ }^{\circ} \mathrm{C}$ to $2 \times 10^{-3} /{ }^{\circ} \mathrm{C}$ (in absolute value) for both horizontal and vertical polarizations and all incidence angles between $0^{\circ}$ and $60^{\circ}$. Recent measurements that were performed by Blanch and Aguasca at the University of Catalonia and reported in [38] and [39] suggest that, for seawater with an SSS of 37.5 psu, the nadir emission brightness temperatures may differ from those predicted by the Klein and Swift model by up to $0.5 \mathrm{~K}$ for a wide range of water temperatures.

Despite the sensitivity of emission brightness temperatures to SSS, SST, and the dielectric model, the impact of dielectric constant variations on scattered celestial noise is expected to be negligible for ocean salinity retrieval. With the exception of some very localized bright spots in the sky (e.g., Cassiopeia A, Orion A, Cygnus A, and Taurus A) for which the downwelling signal can reach extreme values greater than $1000 \mathrm{~K}$ [40], [41], the celestial brightness temperature at L-band varies from $2.75 \mathrm{~K}$ to about $10 \mathrm{~K}$ [3]. Therefore, the sensitivity of the specularly reflected celestial brightness temperatures to SSS and 
SST might approach $0.035 \mathrm{~K} / \mathrm{psu}$ and $0.02 \mathrm{~K} /{ }^{\circ} \mathrm{C}$, respectively. These values are representative of a worst case scenario since the surface reflectivity significantly drops in the presence of roughness. Likewise, the impact of the dielectric model should be no more than about $0.02 \mathrm{~K}$. As the focus of this paper is the angular spreading effect of the surface roughness, the use of any one of the several available dielectric models at L-band is reasonable. For the results that follow, we evaluated the scattering cross sections at an SST of $15{ }^{\circ} \mathrm{C}$ and an SSS of 35 psu.

\section{Representation of the SCATtered Celestial RADiATION Signal at THE SURFACE}

As previously mentioned, to obtain the total scattered signal at the surface $\tilde{T}_{p}^{g s}$ in a given direction $\left(\theta_{s}, \phi_{s}\right)$, we must integrate the brightness temperature contributions from waves incident at the target from all directions over the upper hemisphere. We simplify the notation and let $\tilde{T}_{p}^{g s}\left(\theta_{s}, \phi_{s}, u_{10}, \varphi_{w}\right) \rightarrow$ $T_{p}^{g s}\left(\theta_{s}, \phi_{s}, u_{10}, \varphi_{w}^{\prime}\right)$, where $\varphi_{w}^{\prime}=\varphi_{w}-\phi_{s}$ is the downwind direction $\varphi_{w}$ relative to the radiometer azimuth $\phi_{s}$. Since the distribution of incident celestial radiation over the upper hemisphere is a function of time and target position on Earth, the rough surface scattered signal at polarization $p$, i.e., $T_{p}^{g s}$, is also a function of latitude $\vartheta_{g}$, longitude $\varphi_{g}$, and time $t$, so that

$$
T_{p}^{g s}=T_{p}^{g s}\left(\vartheta_{g}, \varphi_{g}, t, \theta_{s}, \phi_{s}, u_{10}, \varphi_{w}^{\prime}\right) .
$$

Although it is natural to express the scattered celestial noise as a function of target location on Earth, time, wind speed and direction, and scattering azimuth and incidence angles, this 7-D representation is both awkward and redundant.

Fortunately, this representation can be simplified. We begin by defining $\left(\alpha_{n}, \delta_{n}\right)$ as the right ascension and declination, respectively, of the unit normal to the horizontal surface at the target. By introducing these two variables, we can remove the explicit dependence on time and express the scattered celestial noise as a function of six variables: $T_{p}^{g s} \rightarrow$ $\bar{T}_{p}^{g s}\left(\alpha_{n}, \delta_{n}, \theta_{s}, \phi_{s}, u_{10}, \varphi_{w}^{\prime}\right)$. However, even in this form, it is difficult to interpret the effects of variations in scattering incidence and azimuth angles since these parameters affect both the scattering cross sections and the specular sky location. The specular sky location, through its relation to the distribution of noise over the upper hemisphere, is a significant factor in determining the magnitude of the scattered signal for any particular scattering geometry at the target. Unfortunately, specular sky location is not an independent variable in this formulation, and it is difficult to visualize the relationship between target location, scattering angles, time, and specular sky location.

To alleviate these problems, we introduce an alternative 6-D representation in terms of specular right ascension and declination $\left(\alpha_{s}, \delta_{s}\right)$, scattering incidence angle, an angle representing the orientation of the incidence plane in the celestial frame, wind speed, and finally, downwind direction relative to the scattering azimuth. In order to represent the scattering solution in terms of these variables, we must find a map between $\left(\alpha_{s}, \delta_{s}\right)$ and $\left(\alpha_{n}, \delta_{n}\right)$. This map will necessarily involve $\theta_{s}$ and $\phi_{s}$, so that we can write the map as

$$
T:\left(\alpha_{n}, \delta_{n}, \theta_{s}, \phi_{s}\right) \rightarrow\left(\alpha_{s}, \delta_{s}, \theta_{s}, \psi_{u h}\right)
$$

where $\theta_{s}$ is the target incidence angle in the specular direction, and $\psi_{u h}$ is the angle between the incidence plane and the plane containing the celestial polar axis and the specular reflection vector (shifted to the origin of the celestial sphere). Map $T$ rotates the unit normal to the target into the specular direction, and an explicit expression for $\psi_{u h}$ is presented in Appendix A.

Using this transformation, we may reexpress the scattered signal at polarization $p$, i.e., $T_{p}^{g s}$, as follows:

$$
\begin{aligned}
& T_{p}^{g s}\left(\vartheta_{g}, \varphi_{g}, t, \theta_{s}, \phi_{s}, u_{10}, \varphi_{w}^{\prime}\right) \rightarrow \\
& \tilde{T}_{p}^{g s}\left(\alpha_{s}, \delta_{s}, \theta_{s}, \psi_{u h}, u_{10}, \varphi_{w}^{\prime}\right) .
\end{aligned}
$$

In this form, the dependence of the scattered signal upon specular sky location is completely separated from the dependence of the signal upon the scattering cross sections. Moreover, this representation effectively isolates the variables with dominant impacts on the scattered signal (specular sky location, incidence angle, and wind speed) from those with relatively small impacts (orientation angle and relative downwind direction). This functional form is used below to examine the expected variability in the sky glitter contamination.

\section{Angular Spreading of Celestial NOISE By SURFACE ROUGHNESS}

Although the celestial glitter may be expressed as a function of six independent parameters, i.e.,

$$
\tilde{T}_{p}^{g s}=\tilde{T}_{p}^{g s}\left(\alpha_{s}, \delta_{s}, \theta_{s}, \psi_{u h}, u_{10}, \varphi_{w}^{\prime}\right)
$$

the number of independent variables remains large and makes it difficult to evaluate the impact of variations of individual parameters on the glitter. However, we expect that the variation of the glitter with respect to changes in the wind speed, downwind direction, incidence angle, and orientation angle will be smaller when the specular direction is located in an area with uniform celestial brightness than when the specular direction is near a local maximum in brightness (e.g., the galactic equator). To examine this notion, we used the model previously described to compute the scattered celestial radiation at specular points near and far from the galactic equator.

According to both approximate scattering models, the bistatic scattering cross sections must be integrated over a solid angle cap of at least $\Delta \theta \simeq 40^{\circ}$ angular width about the specular direction to account for $90 \%$ of the reflectivity at L-band. Therefore, for "cold" specular points close to the galactic equator, contamination by neighboring bright sources located within $40^{\circ}$ of the specular direction might significantly increase the intensity of the scattered signal. To examine this possibility, we evaluated the scattered signal over the celestial sphere at constant scattering incidence angle $\theta_{s}=0^{\circ}$.

For this analysis, we considered only the zeroth harmonic of the scattered signal, so that wind direction effects were neglected. Moreover, we averaged the scattered signal over all $\psi_{u h}$ to remove the effect of variations in the incidence plane orientation angle. The calculations were performed at wind speeds of 0 (flat surface), 3 , and $10 \mathrm{~m} / \mathrm{s}$. For efficiency, 


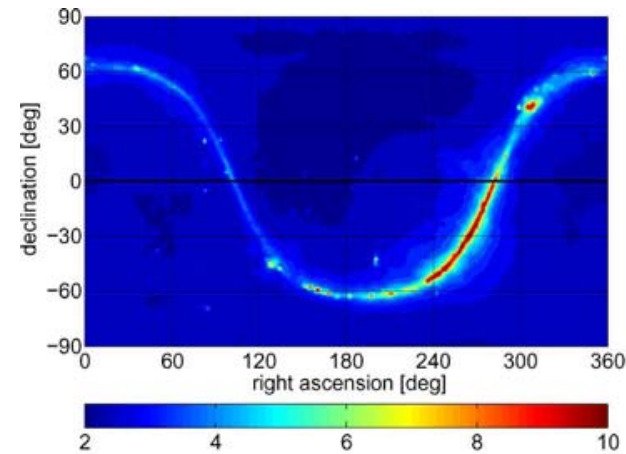

(a)

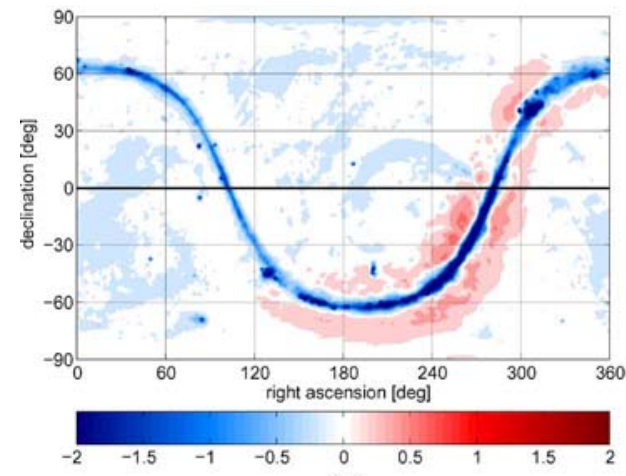

(c)

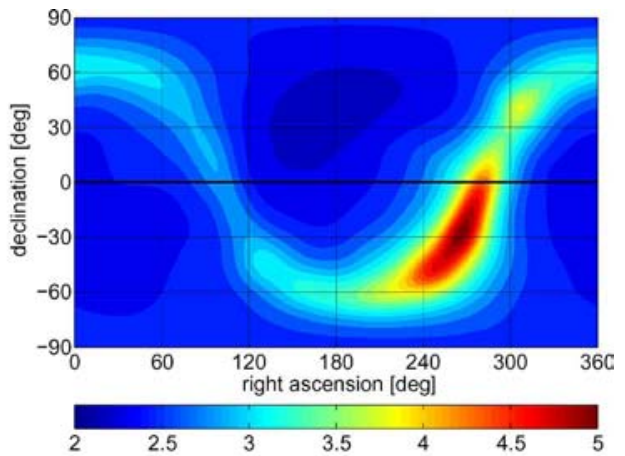

(b)

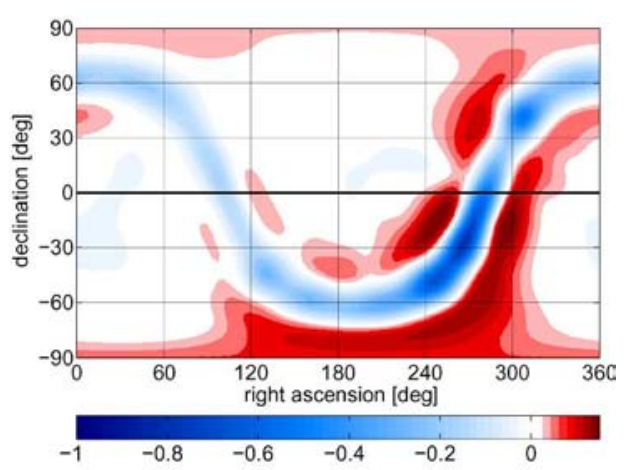

(d)

Fig. 2. Angular spreading of unpolarized celestial noise $\left(\left(T_{h}^{g s}+T_{v}^{g s}\right) / 2\right)$ in the presence of surface roughness at normal incidence. For the rough surface calculations, the results were averaged over all possible orientation angles $\psi_{u h}$ and include only the zeroth harmonic with respect to wind direction. (a) Flat surface specularly reflected signal $\left(\mathrm{SST}=15^{\circ} \mathrm{C}, \mathrm{SSS}=35 \mathrm{psu}\right.$ ). (b) Rough surface scattered signal at $u_{10}=3 \mathrm{~m} / \mathrm{s}$. (c) Difference between the scattered noise at $u_{10}=3 \mathrm{~m} / \mathrm{s}$ and the flat surface reflected noise. (d) Difference between the scattered noise at $u_{10}=10 \mathrm{~m} / \mathrm{s}$ and that at $u_{10}=3 \mathrm{~m} / \mathrm{s}$. All units are kelvin.

the numerical integrations were performed on a $3.75^{\circ} \times 3.75^{\circ}$ grid rather than on the original $0.25^{\circ} \times 0.25^{\circ}$ grid in celestial coordinates. In the next section, we will demonstrate that the use of this reduced-resolution grid has negligible impact on the results.

The results are illustrated in Fig. 2, which shows the differences between the nadir unpolarized scattered celestial noise $\left(T_{h}^{g s}+T_{v}^{g s}\right) / 2$ evaluated for a flat surface and for the two rough surfaces corresponding to wind speeds of 3 and $10 \mathrm{~m} / \mathrm{s}$. The effect of the angular spreading of the scattered signal is evident, particularly in the vicinity of the galactic equator, where the difference between the flat surface reflected signal and the scattered signal at $3 \mathrm{~m} / \mathrm{s}$ reaches nearly $2 \mathrm{~K}$ in magnitude, with a distinct minimum along the equator and maxima on either side. This illustrates the significant spreading effect of the rough surface. Moreover, the solutions at 3 and $10 \mathrm{~m} / \mathrm{s}$ differ by up to $1 \mathrm{~K}$ along the equator and by up to $0.15 \mathrm{~K}$ within a strip extending approximately $30^{\circ}$ on either side of the equator, suggesting that the spreading effect of the rough surface extends the impact of surface roughness well beyond what we expect if the roughness merely reduced reflectivity in the specular direction.

\section{Pointwise AnAlysis of the Rough SEA SuRfaCE EFFECT}

Although the results discussed in the preceding section illustrate the basic tendency of the rough surface to spread the scattered celestial noise about the specular direction, the analysis only applies to a nadir-viewing radiometer, and we did not consider the potential impacts of relative downwind direction $\varphi_{w}^{\prime}$ and the incidence plane orientation angle in the celestial frame. In this section, we address these issues by examining the scattering solutions at "cold" and "hot" points in the sky map as a function of radiometer incidence angle, wind speed, relative downwind direction, and incidence plane orientation angle.

\section{A. Incidence Angle and Wind Speed Dependence at Hot and Cold Points}

For this analysis, we considered two specular locations in the sky, as illustrated by two white dots in Fig. 3(a). One point, which is located at $\left(\alpha_{s}=200^{\circ}, \delta_{s}=60^{\circ}\right)$, is in an area with relatively low and spatially uniform brightness temperature (hereafter referred to as the cold point) around $3.3 \mathrm{~K}$ whereas the other, which is located at $\left(\alpha_{s}=265^{\circ}, \delta_{s}=-32.5^{\circ}\right)$, is close to the galactic plane. Here, there is a local brightness temperature maximum of approximately $15 \mathrm{~K}$ on the $0.25^{\circ} \times$ $0.25^{\circ}$ grid (hereafter referred to as the hot point). Fig. 3(b) shows the full-resolution unpolarized celestial noise map in the vicinity of the hot point.

Having established these specular points, we evaluated $\tilde{T}_{p}^{g s}\left(\alpha_{s}, \delta_{s}, \theta_{s}, \psi_{u h}, u_{10}, \varphi_{w}^{\prime}\right)$ for varying values of $\theta_{s}$ and $u_{10}$ but at a fixed upper hemisphere orientation angle of $\psi_{u h}=0^{\circ}$. For efficiency, we did this using a reduced-resolution celestial noise map, which was derived by averaging the full-resolution map onto the $3.75^{\circ} \times 3.75^{\circ}$ grid introduced in the previous section. This averaging was performed in such a way as to 


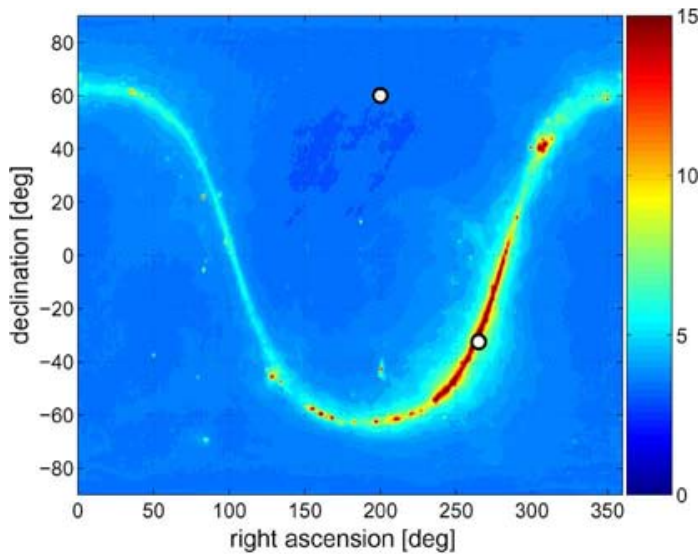

(a)



(c)

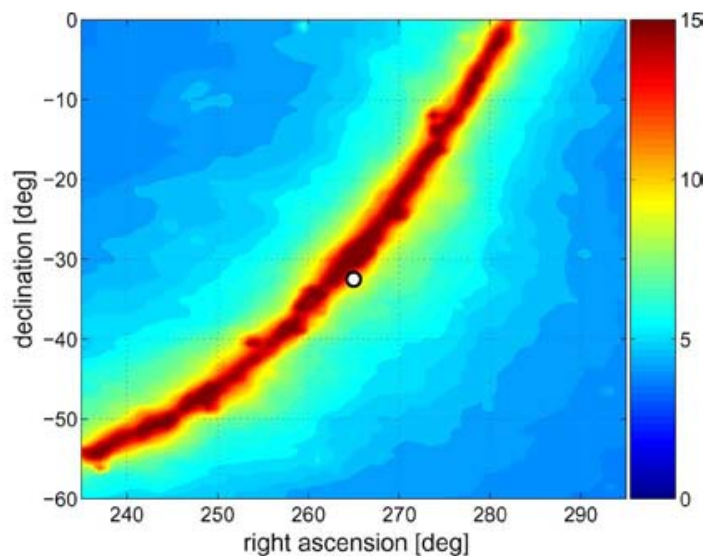

(b)

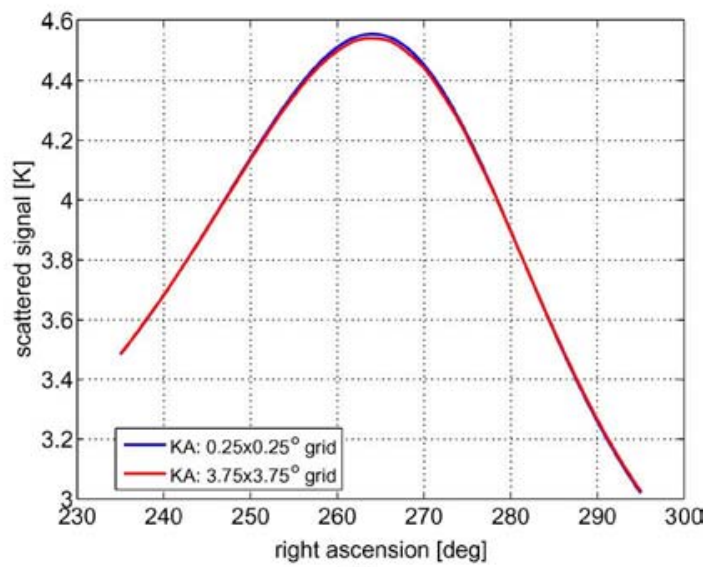

(d)

Fig. 3. (a) Unpolarized celestial noise map in the celestial coordinates. White dots show locations of "hot" and "cold" points where we examine the behavior of the scattered signal with respect to changes in surface roughness and scattering geometry. (b) Zoom on the region around the "hot" point at $\left(\alpha_{s}=265^{\circ}, \delta_{s}=\right.$ $-32.5^{\circ}$ ). (c) Same as (b), except the sky map background is plotted using the reduced-resolution map, with a grid spacing of $3.75^{\circ} \times 3.75^{\circ}$ in celestial coordinates. (d) Scattered celestial noise $\left(T_{h}^{g s}+T_{v}^{g s}\right) / 2$ based on both the (blue) full- and (red) reduced-resolution celestial noise maps. For the scattering calculations, the relative downwind direction $\varphi_{w}^{\prime}=\varphi_{w}-\phi_{s}=0^{\circ}$, the orientation angle $\psi_{u h}=0^{\circ}$, wind speed $u_{10}$ is $7 \mathrm{~m} / \mathrm{s}$, and the incidence angle $\theta_{s}=0^{\circ}$. Brightness temperatures are expressed in kelvin.

preserve the total power integrated over each coarse grid cell. As may be seen in Fig. 3(c), the elongated maximum in brightness temperature is significantly smoothed compared with that in the full-resolution map [Fig. 3(b)].

In order to justify the use of the reduced-resolution map in the practical implementation of the scattering calculation, we had to ensure that the error incurred through the use of this map rather than the full-resolution map is negligible. To do so, we computed, using both the $0.25^{\circ} \times 0.25^{\circ}$ and $3.75^{\circ} \times 3.75^{\circ}$ celestial grids, the scattered celestial noise along constant-declination cross sections through the hot point using the Kirchhoff scattering model and the Kudryavtsev et al. [34] wave spectrum driven by a wind speed of $7 \mathrm{~m} / \mathrm{s}$. Fig. 3(d) shows the nadir unpolarized scattered celestial noise $\left(T_{h}^{g s}+\right.$ $\left.T_{v}^{g s}\right) / 2$ calculated using both grids. The two curves are nearly indistinguishable, suggesting that the use of the coarse grid is acceptable. Therefore, we used the $3.75^{\circ} \times 3.75^{\circ}$ celestial grids for all scattering calculations that follow.

For both the cold and hot spots, we evaluated the scattered signal as a function of incidence angle for the flat sea as well as for seas roughened by the surface wind with speeds of 3, 8, 13, and $18 \mathrm{~m} / \mathrm{s}$. Fig. 4(a) and (b) shows horizontally and vertically polarized celestial glitter at the cold point as a function of incidence angle from $0^{\circ}$ to $60^{\circ}$. At this point, where the incoming celestial noise is uniform in the vicinity of the specular direction, the behavior of the celestial glitter is expected to be consistent with the behavior of reflectivity. As the surface becomes rougher, we expect that the emissivity will increase for both horizontal and vertical polarizations and that (by Kirchhoff's law) the reflectivity will decrease, at least for the incidence angles considered here. Moreover, we expect that the incidence angle should remain the dominant parameter determining the reflected signal, so that the glitter should increase with incidence angle at horizontal polarization and decrease with incidence angle at vertical polarization. According to rough surface emissivity models at L-band [42], [43], we expect a slight decrease (relative to the flat surface values) in the scattered signal at all incidence angles less than about $55^{\circ}$.

Indeed, as expected, the horizontally polarized scattered celestial noise increases with increasing incidence angle [Fig. 4(a)] and is lower than the flat surface values for incidence angles beyond about $35^{\circ}$. The significant (up to $0.15 \mathrm{~K}$ at $60^{\circ}$ incidence angle) decrease in scattered signal in the $3-\mathrm{m} / \mathrm{s}$ 




(a)



(c)

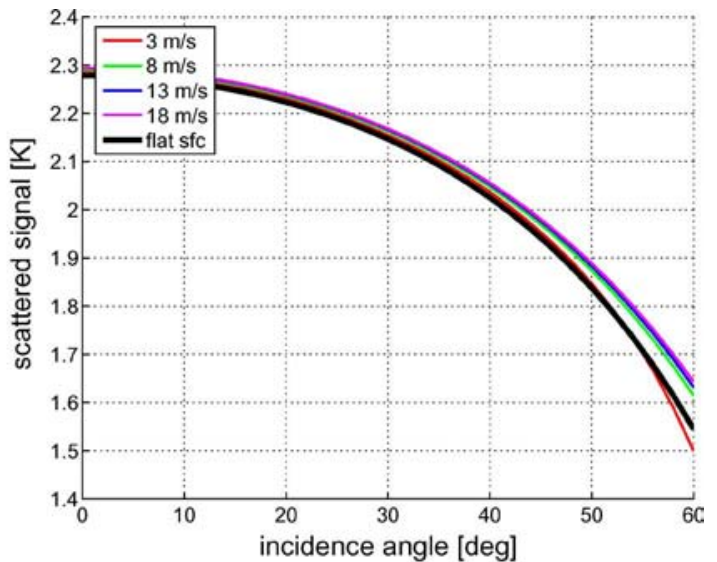

(b)

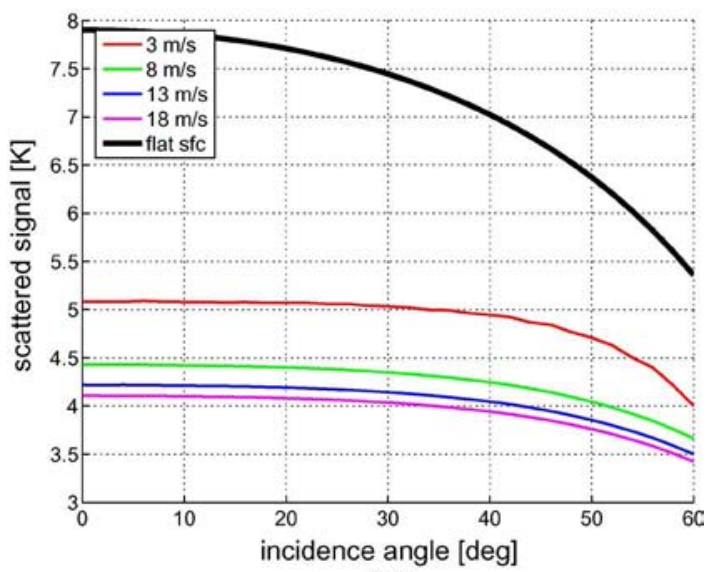

(d)

Fig. 4. (a) Horizontally and (b) vertically polarized scattered celestial noise computed using the Kirchhoff electromagnetic model at the cold specular point located at $\left(\alpha_{s}=200^{\circ}, \delta_{s}=60^{\circ}\right)$ plotted as a function of scattered wave incidence angle for different wind speeds. (c) and (d) Same as in (a) and (b) but for the hot specular point located at $\left(\alpha_{s}=265^{\circ}, \delta_{s}=-32.5^{\circ}\right)$. The orientation angle $\psi_{u h}=0^{\circ}$.

wind speed case beyond $50^{\circ}$ may be numerical in origin since, at such incidence angles, the narrowness of the scattering cross sections at low wind speeds becomes problematic at the grid spacing employed here. Within $35^{\circ}$ of nadir, the rough surface solutions slightly exceed the flat surface values, in contrast to what we expect from rough surface emissivity models. This result suggests that, even at this cold point, stronger celestial noise from distant locations in the celestial sphere may contribute (although weakly) to the total scattered signal.

At vertical polarization and at all wind speeds the glitter decreases with increasing incidence angle [Fig. 4(b)]. As in the case of horizontal polarization at small incidence angles, the rough surface scattered noise is larger than the flat surface counterparts except for the $3-\mathrm{m} / \mathrm{s}$ case at incidence angles beyond $55^{\circ}$.

For both linear polarizations, the differences between flat surface reflected noise and rough surface counterparts never exceed about $10 \%$ of the original signal at the cold point, and they tend to increase with increasing incidence angle. The glitter increases with increasing wind speed but generally remains within $2 \%$ of the flat surface values for incidence angles below $50^{\circ}$. Beyond $50^{\circ}$, the rough surface solutions deviate from flat surface signals by up to about $5 \%$.
At the hot point, several differences from the cold point behavior are worth noting. First, at both linear polarizations [Fig. 4(c) and (d)], the scattered signals are all lower than the perfectly flat surface counterparts, and they decrease with increasing wind speed at all incidence angles and polarizations. Moreover, the relative differences between flat and rough surface scattered signals are much larger than those at the cold point, with differences exceeding $30 \%$ of the flat surface signal at a wind speed of $8 \mathrm{~m} / \mathrm{s}$. Differences between rough and flat surface solutions are nearly independent of polarization and increase with increasing wind speed, reaching a maximum difference of nearly $50 \%$ of the flat surface signal at $18 \mathrm{~m} / \mathrm{s}$. At horizontal polarization, the absolute differences are nearly constant with incidence angle, whereas, at vertical polarization, the absolute differences tend to decrease with increasing incidence angle.

To summarize, for both linear polarizations, the rough surface scattered celestial noise at the cold and homogeneous point generally differs from the corresponding flat surface values by less than $0.05 \mathrm{~K}$, with slightly larger differences at larger wind speeds and, at vertical polarization, at incidence angles beyond about $50^{\circ}$. At the hot point, differences are much larger, with a difference of nearly $3.5 \mathrm{~K}$ between the $8-\mathrm{m} / \mathrm{s}$ and flat surface values near nadir. 


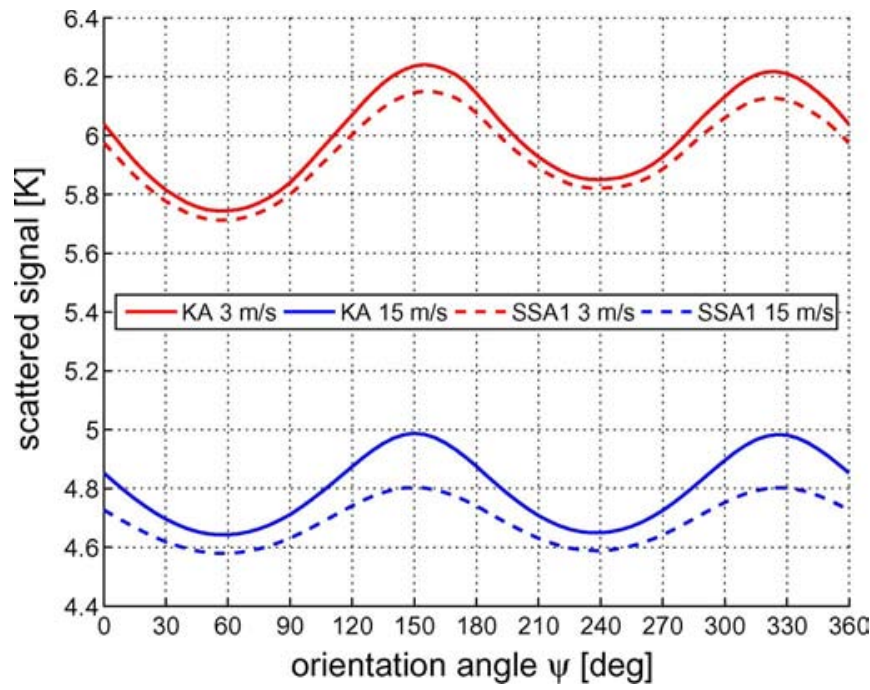

Fig. 5. Scattered horizontally polarized celestial glitter computed using the KA and SSA-1 scattering models at the hot specular point $\left(\alpha_{s}=265^{\circ}\right.$, $\delta_{s}=-32.5^{\circ}$ ) plotted as a function of incidence plane orientation angle $\psi_{u h}$ for both (red) low and (blue) high surface wind speeds. The incidence angle is $40^{\circ}$.

\section{B. Impact of Orientation Angle}

So far, we have considered the scattered signal variability as a function of wind speed and incidence angle but at a fixed upper hemisphere orientation angle $\psi_{u h}=0^{\circ}$. In this section, we consider the impact of the orientation angle at fixed wind speed, incidence angle, and specular sky location.

The orientation angle dependence is a strong function of incidence angle. At nadir, we expect a small impact of the orientation angle parameter, owing to both the fact that the portion of the sky contributing to the scattered signal is not a function of the orientation angle and the fact that, at nadir, the scattering cross sections are nearly symmetric about the specular direction. Scattering calculations (not shown) reveal almost no impact of the orientation angle at the cold point and a negligible dependence of less than $0.05 \mathrm{~K}$ for the hot point at nadir, regardless of the type of scattering model, polarization, or wind speed.

At the hot point, the situation is quite different. Fig. 5 shows the symmetric (i.e., with no wind direction dependence) scattered horizontally polarized signal at an incidence angle of $40^{\circ}$ as a function of the orientation angle obtained using both scattering models at wind speeds of 3 and $15 \mathrm{~m} / \mathrm{s}$. The peakto-peak amplitude of the variation is about ten times larger than that at nadir, with an amplitude on the order of $0.5 \mathrm{~K}$ at $3 \mathrm{~m} / \mathrm{s}$ and $0.3 \mathrm{~K}$ at $15 \mathrm{~m} / \mathrm{s}$ for both electromagnetic models and linear polarizations (not shown). These results should be anticipated since, far from nadir, the scattering cross sections become elongated in the incidence plane, and the dominant source of variation with $\psi_{u h}$ is the variation of the orientation of this elongated cross section maximum on the celestial sphere.

In order to provide an indication of the variability of the orientation angle sensitivity over the entire celestial sphere, in Fig. 6, we display the peak-to-peak amplitude of the variation of the horizontally polarized scattered celestial noise at two incidence angles. Fig. 6(a) shows that, at nadir and at a wind speed of $7 \mathrm{~m} / \mathrm{s}$, the amplitude is generally maximum near the galactic equator and reaches approximately $0.01 \mathrm{~K}$. At an incidence angle of $20^{\circ}$ (not shown), the amplitudes are also maximum near the galactic equator but are nearly an order of magnitude larger than those at nadir, reaching just over $0.1 \mathrm{~K}$. At an incidence angle of $40^{\circ}$, as shown in Fig. 6(b), the maximum peak-to-peak amplitude is nearly $0.4 \mathrm{~K}$, and secondary peaks on either side of the galactic equator are evident. At a wind speed of $25 \mathrm{~m} / \mathrm{s}$ (not shown), amplitudes are slightly less than those at $7 \mathrm{~m} / \mathrm{s}$ but are still larger at an incidence angle of $40^{\circ}$ than at $20^{\circ}$.

The secondary maxima may be explained as follows. When the specular point is very near the galactic equator, we expect a maximum scattered signal when the incidence plane is aligned with the galactic plane and a minimum signal when the incidence plane is orthogonal to the galactic plane. When the specular point is located far away from the galactic plane, we expect the opposite situation, with maximum scattered signal when the incidence plane is orthogonal to the galactic plane so that the maxima in the scattering cross sections intersect the galactic plane. At some intermediate specular point between these two extremes, we expect that, as the incidence plane orientation angle varies through $360^{\circ}$, the integrated product of the scattering cross sections and the celestial noise will remain nearly constant.

Overall, the impact of orientation angle is negligible at low incidence angles for all specular points including those in the vicinity of the galactic equator. For incidence angles beyond about $20^{\circ}$, the impact becomes nonnegligible for ocean salinity retrieval in the vicinity of the galactic plane.

\section{Impact of Wind Direction}

Sea surface emissivity models at L-band predict a weak but definite dependence of emission on the angle between the wind and emission directions [44]. The surface directional spectrum, as derived from the Fourier transform of the sea surface elevation covariance function, exhibits no odd azimuthal harmonics. As shown in [42], there is a direct correspondence between emission and surface azimuthal harmonics; thus, sea surface emissivities and reflectivities evaluated with such models exhibit only even azimuthal harmonics with respect to relative wind direction. Moreover, rough surface directional spectra are typically symmetric about the downwind direction, so that both the horizontally and vertically polarized emissivities and reflectivities must be symmetric about the downwind direction [45]. Downwelling atmospheric radiation and attenuation can significantly modify these azimuthal signatures, as found (at microwave frequencies of above $18 \mathrm{GHz}$ ) in [46] using a geometric optics model and in [47] using a two-scale model. Depending on the specular location in the sky, downwelling celestial radiation can have a significantly different effect than downwelling atmospheric radiation.

In Fig. 7(a), we show the predicted amplitudes of the surface emission second azimuthal harmonics at horizontal and vertical polarizations as a function of wind friction velocity $u_{*}$, using the small slope approximation/small perturbation method (SSA/SPM) emissivity model [42], [43] evaluated at incidence 


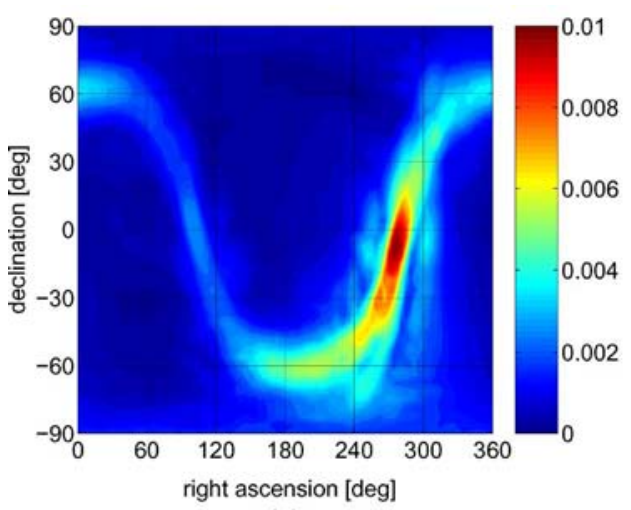

(a)

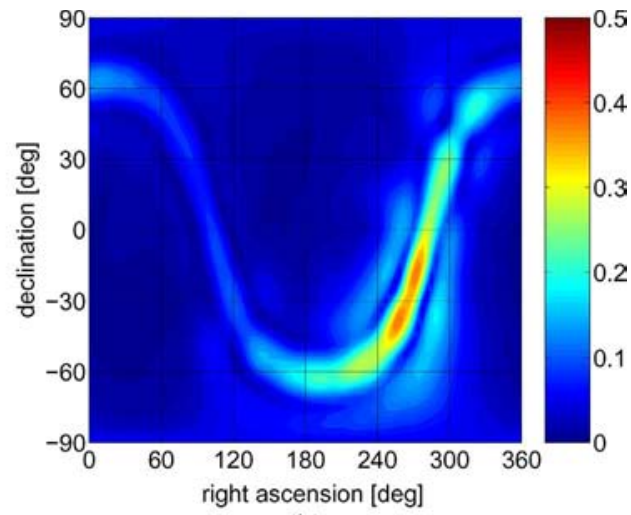

(b)

Fig. 6. Peak-to-peak amplitudes of the isotropic (i.e., the wind direction is independent) component of the scattered horizontally polarized celestial glitter evaluated over all orientation angles $\psi_{u h}$ using the KA scattering model at a wind speed of $7 \mathrm{~m} / \mathrm{s}$ and (a) at nadir and (b) at an incidence angle of $40^{\circ}$.

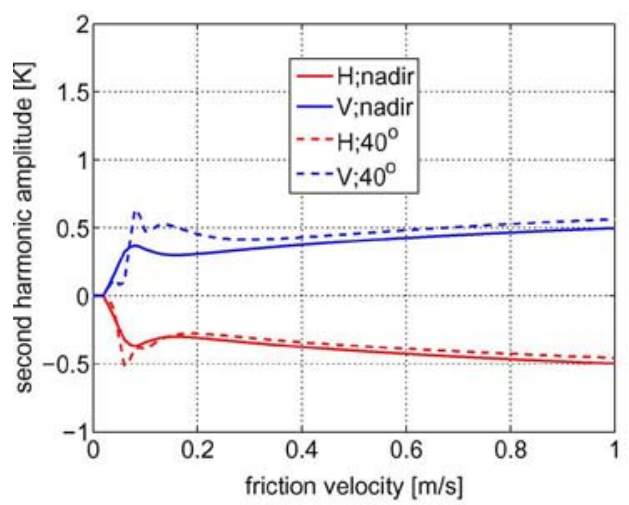

(a)

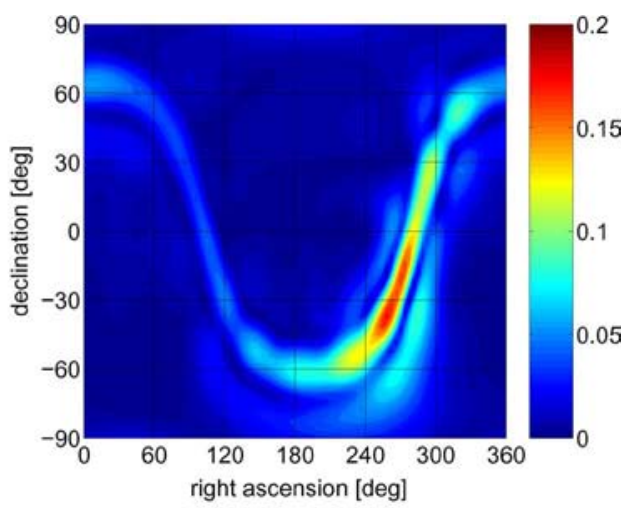

(b)

Fig. 7. (a) Second-harmonic ( $\left.\cos 2\left(\varphi_{w}-\phi_{s}\right)\right)$ amplitudes of rough surface emission at (red) horizontal and (blue) vertical polarizations, as obtained from the SSA/SPM model (solid line) at nadir and (dashed line) at an incidence angle of $40^{\circ}$. (b) Second-harmonic amplitude of the horizontally polarized scattered celestial noise evaluated with the KA model at $\psi_{u h}=0^{\circ}$ and at an incidence angle of $40^{\circ}$. Both the emission and scattered celestial noise harmonics are evaluated at a surface wind speed of $7 \mathrm{~m} / \mathrm{s}$, an SST of $15^{\circ} \mathrm{C}$, and an SSS of $35 \mathrm{psu}$.

angles of $0^{\circ}$ and $40^{\circ}$ using the Kudryavtsev et al. [34] spectral model at an SST of $15^{\circ} \mathrm{C}$ and an SSS of 35 psu. As shown, the dependence on friction velocity and incidence angle is rather weak, with a nearly constant harmonic amplitude of about $0.5 \mathrm{~K}$ (or $1 \mathrm{~K}$ peak-to-peak amplitude) in vertical polarization and $-0.5 \mathrm{~K}$ in horizontal polarization (i.e., with a phase difference of $180^{\circ}$ between them).

As detailed in Appendix B and in [44], the azimuthal harmonic expansion of the bistatic scattering cross sections up through second order $[m=0,1$ in (18)] yields the following azimuthal harmonic expansion of the total scattered celestial noise signal at polarization $p$ :

$$
\begin{aligned}
\tilde{T}_{p}^{g s}\left(\alpha_{s}, \delta_{s}, \theta_{s}, \psi_{u h}, u_{10}, \varphi_{w}^{\prime}\right)= & \tilde{A}_{p}^{(0)}\left(\alpha_{s}, \delta_{s}, \theta_{s}, \psi_{u h}, u_{10}\right) \\
& +\tilde{A}_{p}^{(2)}\left(\alpha_{s}, \delta_{s}, \theta_{s}, \psi_{u h}, u_{10}\right) \\
& \times \cos \left(2 \varphi_{w}^{\prime}\right) \\
& +\tilde{B}_{p}^{(2)}\left(\alpha_{s}, \delta_{s}, \theta_{s}, \psi_{u h}, u_{10}\right) \\
& \times \sin \left(2 \varphi_{w}^{\prime}\right)
\end{aligned}
$$

In contrast to surface emission models, spatial heterogeneity in the celestial radiation sources induces a nonzero amplitude $\tilde{B}_{p}^{(2)}$ of the $\sin \left(2 \phi_{w}^{\prime}\right)$ component of the scattered celestial noise. In
Fig. 7(b), we show the scattered signal second-harmonic amplitude at $\psi_{u h}=0^{\circ}$ for horizontal polarization computed using the KA scattering model at an incidence angle of $40^{\circ}$ and a wind speed of $7 \mathrm{~m} / \mathrm{s}$. The amplitudes reach approximately $0.1-0.2 \mathrm{~K}$, tend to achieve maxima along the galactic equator, and do not exhibit a strong dependence on incidence angle or wind speed. The source of the secondary maxima in the azimuthal harmonic amplitude on either side of the galactic plane is analogous to that of the secondary peaks in the orientation angle dependence. Unlike surface emission, the phase

$$
\Phi\left(\alpha_{s}, \delta_{s}, \theta_{s}, \psi_{u h}, u_{10}\right)=\tan ^{-1}\left(\frac{-\tilde{B}_{p}^{(2)}}{\tilde{A}_{p}^{(2)}}\right)
$$

is a function of specular location in the sky, incidence angle, wind speed, and incidence plane orientation angle. As found (not shown), the phase varies by nearly $180^{\circ}$ as the specular point shifts across the galactic equator in some portions of the celestial sphere. Similar patterns are observed over a range of incidence angles and wind speeds, suggesting that the main factor determining this phase angle is the specular location in the sky.

Although incidence angle has little impact on the phase angle at a fixed specular location, it is important to appreciate that, 


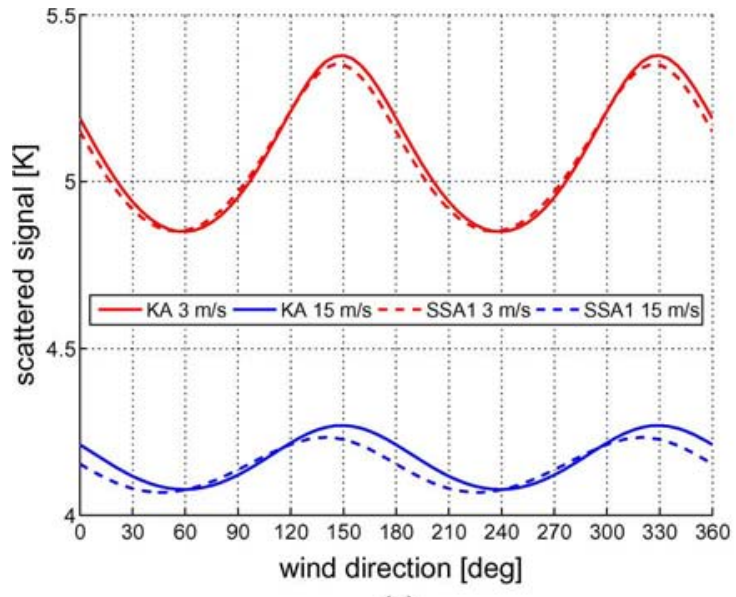

(a)

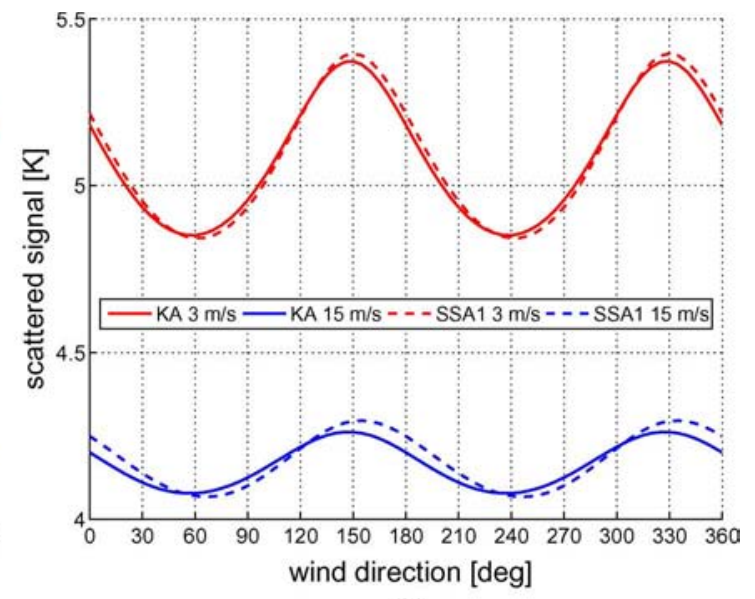

(b)

Fig. 8. Horizontally and vertically polarized scattered celestial noise computed using the KA and the SSA-1 scattering models at the hot specular point located at

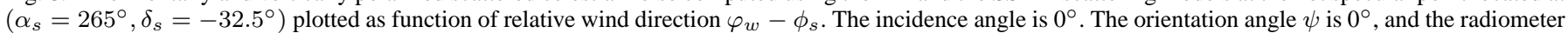
azimuth angle is $0^{\circ}$. (a) $\mathrm{H}$ polarization: incidence angle $0^{\circ}$. (b) $\mathrm{V}$ polarization: incidence angle is $0^{\circ}$.

in many cases (such as aircraft-based radiometry), the Earth target is fixed, and the radiometer azimuth varies at a (roughly) fixed incidence angle. For incidence angles far away from nadir, small variations in azimuth can lead to large changes in this phase angle, owing to changes in the specular location at a fixed Earth target. This complicates the interpretation of azimuthal signatures in aircraft radiometer measurements.

In order to compare the impacts of relative wind azimuth and orientation angle, we computed the scattered noise for the full range of relative wind directions at the same hot and cold specular points previously considered. At the cold point, the impact of the relative wind direction is negligible (i.e., less than $0.05 \mathrm{~K}$ ) for both scattering models at all incidence angles, regardless of wind speed.

At the hot point (see Fig. 8) and with an incidence of $0^{\circ}$, the KA and the SSA-1 solutions are nearly in phase at horizontal and vertical polarizations. Moreover, both models predict peakto-peak amplitudes of approximately $0.5 \mathrm{~K}$ at a wind speed of $3 \mathrm{~m} / \mathrm{s}(0.25 \mathrm{~K}$ at $15 \mathrm{~m} / \mathrm{s})$, which is about 100 times larger than that at the cold point. Unlike the emission predictions of the SSA/SPM emissivity model, there is little difference between the phases of the solutions for the two linear polarizations. To understand this, we note that the variation in scattered signal with relative wind direction has two components: one involves a variation in the reflectivity (scattering cross sections integrated over the entire upper hemisphere), and the other involves a rotation of the nonuniform cross sections about the specular direction. For both scattering models, the phases of the relative wind direction reflectivity harmonics differ by $180^{\circ}$ between horizontal and vertical polarizations. However, the azimuthal structure of the total $\left(\sigma_{p p}+\sigma_{p q}\right)$ cross sections about the specular direction is similar for both polarizations, regardless of the scattering model used. Therefore, for a relatively uniform source, where the first effect dominates, the two polarizations should be out of phase, whereas, for a strongly nonuniform source, where the second effect dominates, the two polarizations should be in phase. In [48], it is demonstrated that, for a nadir-viewing instrument, the orthogonal brightness temperature components of ocean surface emission must ex- hibit a $\cos 2\left(\varphi_{w}-\phi_{r}\right)$ wind direction dependence, where $\phi_{r}$ is the radiometer viewing azimuth. In the presence of scattered nonuniform sky radiation, these brightness temperature components must still exhibit this functional dependence at nadir, but unlike the situation without nonuniform downwelling radiation, here, a change in the absolute wind direction is not equivalent to a rotation of the instrument polarization ports.

At an incidence angle of $40^{\circ}$ at the hot point, amplitudes and phases are similar to those at nadir, with peak-to-peak amplitudes near $0.7 \mathrm{~K}$ at horizontal polarization and $0.4 \mathrm{~K}$ at vertical polarization for both scattering models for a wind speed of $3 \mathrm{~m} / \mathrm{s}$. At $40^{\circ}$, there is a small but noticeable offset between the KA and the SSA-1 at both polarizations, with the SSA-1 solution consistently lower (larger) than that of the KA for all wind directions at the $\mathrm{H}(\mathrm{V})$ polarization at both 3 and $15 \mathrm{~m} / \mathrm{s}$.

Overall, the relative wind direction dependence is similar in magnitude to the orientation angle dependence, with peak-topeak amplitudes ranging from negligible away from the galactic plane to about $0.7 \mathrm{~K}$ at horizontal polarization at an incidence angle of $40^{\circ}$ near the galactic equator. In comparison with the orientation angle dependence, the wind direction dependence is more uniform with the incidence angle, which follows from the fact that the wind direction dependence stems mainly from anisotropy in the rough surface rather than anisotropy in the scattering cross sections about the specular point. It is important to note that the wind direction results presented here are strongly dependent on the azimuthal spreading function of the surface wave model and that alternative surface wave models may yield significantly different, and possibly much lower, amplitudes.

\section{CONCLUSION}

Scattering of incoming celestial radiation by the roughened ocean surface presents a significant challenge for an operational ocean surface salinity retrieval algorithm. The preceding results suggest that the errors associated with assuming that the celestial contribution to antenna temperature may be computed via flat surface Fresnel reflection coefficients may far exceed an 
acceptable threshold of $0.1 \mathrm{~K}$, even for specular points of up to $20^{\circ}$ away from the galactic equator. A particularly important aspect of the problem is that, by the very nature of celestial radiation, these errors are likely to introduce consistent regional and seasonal biases in antenna temperature. Moreover, the angular spreading effect of the rough surface, as illustrated in Fig. 2, suggests that the use of a simple wind-speed-dependent reduction of the flat surface reflectivity is unlikely to satisfactorily reduce these systematic errors in the vicinity of the galactic equator.

One way to address the problem is to precompute scattered celestial noise for an appropriate range of surface conditions and viewing geometries. With this strategy, care must be taken to express the solution in an efficient manner. In this paper, we have simplified the problem by neglecting atmospheric attenuation and Faraday rotation. With these simplifications and for the chosen surface description [34], the scattered celestial noise may be expressed as a function of six variables: specular right ascension and declination, scattering incidence angle, incidence plane orientation angle $\psi_{u h}, 10 \mathrm{~m}$ wind speed, and wind direction relative to the scattering azimuth.

Based on the calculations presented here, none of these variables may be neglected. The calculations suggest that sensitivity of orthogonal channel brightness temperatures to the orientation angle may reach $0.5 \mathrm{~K}$ near the galactic equator for incidence angles beyond $40^{\circ}$. Variations with the relative wind direction may exceed this and may reach approximately $0.5 \mathrm{~K}$ even for nadir viewing. Moreover, the phases of the relative wind direction harmonics may strongly depend on specular location in the sky.

The wind speed dependence is not negligible even at high wind speeds. Given that the sensitivity of L-band brightness temperature to salinity ranges from about 0.2 to $0.8 \mathrm{~K} / \mathrm{psu}$, even the $0.2-\mathrm{K}$ decrease in scattered signal between 8 and $13 \mathrm{~m} / \mathrm{s}$ observed at the hot point is significant, corresponding to a 1-psu difference in the SSS at worst.

Errors in this celestial glint model are certainly expected due to 1) errors in the sky map (including polarization); 2) inaccuracies in the statistical description of the rough sea surface; and 3) validity of the asymptotic scattering models. Polarized L-band sky data presented in [49] suggest that the maximum polarized power of the incoming celestial noise should not exceed about $0.5 \mathrm{~K}$. Based on this information and the similarity of the results obtained with the KA and the SSA-1 electromagnetic models, we anticipate that the dominant source of error is the inaccuracies in the statistical description of the rough sea surface and that this error may alter both the isotropic and harmonic components of the scattered noise.

The rough surface impact on scattered celestial noise is mostly attributable to the angular spreading of the quasispecular lobe, and this spreading is primarily associated with the growth of the ocean surface wave MSS with increasing wind speed. Effective MSSs retrieved from global positioning system L-band bistatic data (e.g., [50]) tend to support the robustness of sea surface spectral models for which the MSS parameter is constrained to agree with the Cox and Munk [35] results. Nevertheless, dedicated validation exercises using real radiometric data are required.
Finally, in this paper, we have considered general properties of the scattered celestial noise apart from any specific mission concept. Considering the geometry of the problem and the strong dependence of the rough surface impact on specular sky location, the extent and pattern of the ultimate impact on the measurements obtained with a particular instrument will strongly depend on the satellite orbital characteristics and viewing geometry. Moreover, given the seasonal dependence of the celestial noise contamination for any sun-synchronous mission (as noted in [3]), it is expected that there will be a significant annual cycle in the rough surface effects at a given location on Earth. In Part II [52], we consider the specific case of SMOS and examine the extent and seasonality of the rough surface influence on the contamination.

\section{APPENDIX A \\ COMPUTATION OF ORIENTATION ANGLE $\psi_{u h}$}

Orientation angle $\psi_{u h}$ must be defined to allow construction of an inverse map $T^{-1}$ that uniquely maps a specular direction $\left(\alpha_{s}, \delta_{s}\right)$ into a target unit normal $\left(\alpha_{n}, \delta_{n}\right)$. To facilitate a definition of $\psi_{u h}$, we first establish basis vectors normal to the line of sight in the specular direction in both the target and celestial frames. These basis vectors are analogous to horizontal and vertical polarization basis vectors used to describe electromagnetic plane waves. In the target frame, which is the topocentric frame whose origin is the surface target, we define the "horizontal" basis vector $\hat{\mathbf{h}}^{\mathbf{u}}=\hat{\mathbf{z}}_{\mathbf{e}} \times \hat{\mathbf{r}} /\left\|\hat{\mathbf{z}}_{\mathbf{e}} \times \hat{\mathbf{r}}\right\|$, where $\hat{\mathbf{z}}_{\mathbf{e}}$ is the unit normal to the surface at the target and $\hat{\mathbf{r}}$ is directed outward toward the specular direction from the target. Next, we define a "vertical" basis vector by $\hat{\mathbf{v}}^{\mathbf{u}}=\hat{\mathbf{r}} \times \hat{\mathbf{h}}^{\mathbf{u}}$. If we let $\phi_{s}^{u}$ and $\theta_{s}^{u}$ be the specular azimuth and altitude, respectively, of $\hat{\mathbf{r}}$ in the target frame, then we have

$$
\begin{aligned}
& \hat{\mathbf{h}}^{\mathbf{u}}=-\sin \phi_{s}^{u} \hat{\mathbf{x}}^{\mathbf{u}}+\cos \phi_{s}^{u} \hat{\mathbf{y}}^{\mathbf{u}} \\
& \hat{\mathbf{v}}^{\mathbf{u}}=-\cos \phi_{s}^{u} \sin \theta_{s}^{u} \hat{\mathbf{x}}^{\mathbf{u}}-\sin \phi_{s}^{u} \sin \theta_{s}^{u} \hat{\mathbf{y}}^{\mathbf{u}}+\cos \theta_{s}^{u} \hat{\mathbf{z}}^{u}
\end{aligned}
$$

where $\hat{\mathbf{x}}^{\mathbf{u}}, \hat{\mathbf{y}}^{\mathbf{u}}$, and $\hat{\mathbf{z}}^{\mathbf{u}}$ are Cartesian basis vectors for the target frame. Analogous basis vectors may be defined in the celestial frame as

$$
\begin{aligned}
& \hat{\mathbf{h}}^{\mathbf{c}}=-\sin \alpha_{s} \hat{\mathbf{x}}^{\mathbf{c}}+\cos \alpha_{s} \hat{\mathbf{y}}^{\mathbf{c}} \\
& \hat{\mathbf{v}}^{\mathbf{c}}=-\cos \alpha_{s} \sin \delta_{s} \hat{\mathbf{x}}^{\mathbf{c}}-\sin \alpha_{s} \sin \delta_{s} \hat{\mathbf{y}}^{\mathbf{c}}+\cos \delta_{s} \hat{\mathbf{z}}^{\mathbf{c}}
\end{aligned}
$$

where $\alpha_{s}$ and $\delta_{s}$ are the specular right ascension and declination, respectively, of $\hat{\mathbf{r}}$ in the celestial coordinate system.

If we denote the components of a vector normal to the line of sight in the $\left(\hat{\mathbf{h}}^{\mathbf{u}}, \hat{\mathbf{v}}^{\mathbf{u}}\right)$ basis by $\left(V^{h u}, V^{v u}\right)^{T}$, then its components in the celestial basis $\left(\hat{\mathbf{h}}^{\mathbf{c}}, \hat{\mathbf{v}}^{\mathbf{c}}\right)$ denoted by $\left(V^{h c}, V^{v c}\right)$ are

$$
\left(\begin{array}{l}
V^{h c} \\
V^{v c}
\end{array}\right)=\left(\begin{array}{cc}
\hat{\mathbf{h}}^{\mathbf{c}} \cdot \hat{\mathbf{h}}^{\mathbf{u}} & \hat{\mathbf{h}}^{\mathbf{c}} \cdot \hat{\mathbf{v}}^{\mathbf{u}} \\
\hat{\mathbf{v}}^{\mathbf{c}} \cdot \hat{\mathbf{h}}^{\mathbf{u}} & \hat{\mathbf{v}}^{\mathbf{c}} \cdot \hat{\mathbf{v}}^{\mathbf{u}}
\end{array}\right)\left(\begin{array}{l}
V^{h u} \\
V^{v u}
\end{array}\right) .
$$

The preceding matrix is a rotation matrix that rotates the vector components $\left(V^{h u}, V^{v u}\right)^{T}$ by an angle $\psi_{u h}$, which is analogous 
to the Claassen angle in radiometry [22]. It is the angle by which one must rotate the vector components defined in the target frame basis $\left(\hat{\mathbf{h}}^{\mathbf{u}}, \hat{\mathbf{v}}^{\mathbf{u}}\right)$ counterclockwise about the line of sight in the specular direction to obtain the vector components in the celestial basis $\left(\hat{\mathbf{h}}^{\mathbf{c}}, \hat{\mathbf{v}}^{\mathbf{c}}\right)$. An explicit expression for this angle is

$$
\psi_{u h}=\tan ^{-1}\left(\frac{-\hat{\mathbf{h}}^{\mathbf{c}} \cdot \hat{\mathbf{v}}^{\mathbf{u}}}{\hat{\mathbf{h}}^{\mathbf{c}} \cdot \hat{\mathbf{h}}^{\mathbf{u}}}\right)
$$

where $\tan ^{-1}()$ is the four-quadrant arctangent function. In implementing the preceding inner products, the components of the basis vectors $\hat{\mathbf{h}}^{\mathbf{u}}$ and $\hat{\mathbf{v}}^{\mathbf{u}}$ may be transformed into the celestial coordinate system by applying the transformation

$$
\begin{aligned}
\tilde{\mathbf{h}}^{\mathbf{u}} & =\mathbf{T}_{\mathbf{a c}} \hat{\mathbf{h}}^{\mathbf{u}} \\
\tilde{\mathbf{v}}^{\mathbf{u}} & =\mathbf{T}_{\mathbf{a c}} \hat{\mathbf{v}}^{\mathbf{u}}
\end{aligned}
$$

where $\tilde{\mathbf{h}}^{\mathbf{u}}$ and $\tilde{\mathbf{v}}^{\mathbf{u}}$ are the basis vectors $\hat{\mathbf{h}}^{\mathbf{u}}$ and $\hat{\mathbf{v}}^{\mathbf{u}}$ but with components expressed in the celestial coordinate system. $\mathbf{T}_{\mathbf{a c}}$ is the transformation matrix from the Earth target frame $\mathcal{A}$ to the celestial true-of-date frame $\mathcal{C}$ [51]. The target frame depends on the location of the target on the surface of the Earth, so that $\mathcal{A}=$ $\mathcal{A}\left(\vartheta_{g}, \varphi_{g}\right)$, where $\vartheta_{g}$ and $\varphi_{g}$ are the geodetic latitude and longitude of the target, respectively. Transformation $\mathbf{T}_{\mathbf{a c}}$ may be expressed as a composite transformation from $\mathcal{A}$ to Earth fixed frame $\mathcal{E}$ and from the Earth fixed frame to celestial frame $\mathcal{C}$, i.e.,

$$
\mathbf{T}_{\mathbf{a c}}\left(\vartheta_{g}, \varphi_{g}, t\right)=\mathbf{T}_{\mathbf{e c}}(H) \mathbf{T}_{\mathbf{a e}}\left(\vartheta_{g}, \varphi_{g}\right)
$$

where $\mathbf{T}_{\mathbf{e c}}$, i.e., the transformation from $\mathcal{E}$ to $\mathcal{C}$, is obtained by a rotation about the Earth axis by hour angle $H$, which in turn is the sum of Greenwich sidereal angle $G$ and nutation angle $\mu$ [51], i.e.,

$$
\mathbf{T}_{\mathrm{ec}}=\mathbf{R}_{\mathbf{z}}(-H)=\mathbf{R}_{\mathbf{z}}(-G-\mu) .
$$

The composite transformation $\mathbf{T}_{\mathbf{a e}}$ from $\mathcal{A}$ to $\mathcal{E}$ is obtained by

$$
\mathbf{T}_{\mathbf{a e}}\left(\vartheta_{g}, \varphi_{g}\right)=\mathbf{R}_{\mathbf{z}}\left(-\varphi_{g}-\frac{\pi}{2}\right) \mathbf{R}_{\mathbf{x}}\left(\vartheta_{g}-\frac{\pi}{2}\right) .
$$

In (A8) and (A9), matrices $\mathbf{R}_{\mathbf{x}}$ and $\mathbf{R}_{\mathbf{z}}$ express coordinate system rotations about the $x$ - and $z$-axes, respectively, in a counterclockwise direction when looking toward the origin.

\section{APPENDIX B}

\section{SCATtered Noise AZIMUThal Harmonics}

Recall that the bistatic scattering cross sections have the form [see (18)]

$$
\begin{aligned}
\sigma_{\alpha \alpha_{\circ}}\left(\theta_{\circ}, \phi_{\circ}, \theta_{s}, \phi_{s}, u_{10}, \varphi_{w}\right)=\sum_{m=0}^{\infty} \sigma_{\alpha \alpha_{\circ}}^{(2 m)} \\
\quad \times\left(\theta_{\circ}, \phi_{s}-\phi_{\circ}, \theta_{s}, u_{10}\right) \cos 2 m\left(\Phi_{s i}-\varphi_{w}\right)
\end{aligned}
$$

where $\theta_{\circ}$ and $\phi_{\circ}$ are the incident wave incidence and azimuth angles, respectively; $\theta_{s}$ and $\phi_{s}$ are the scattered wave incidence and azimuth angles, respectively; and $u_{10}$ and $\varphi_{w}$ are the $10-\mathrm{m} / \mathrm{s}$ wind speed and direction, respectively. $\Phi_{s i}$ is the azimuth angle of the difference between the scattered and incident wave vectors. As amplitudes for harmonics greater than two have at most one-tenth the magnitude of the second harmonic, hereafter, we only consider the zeroth and second harmonics.

Retaining only the zeroth and second harmonics and factoring out wind direction $\varphi_{w}$ from the preceding expression, we obtain

$$
\begin{aligned}
& \sigma_{\alpha \alpha_{\circ}}\left(\theta_{\circ}, \phi_{\circ}, \phi_{s}, \theta_{s}, u_{10}, \varphi_{w}\right) \\
& =\sigma_{\alpha \alpha_{\circ}}^{(0)}\left(\theta_{\circ}, \phi_{s}-\phi_{\circ}, \theta_{s}, u_{10}\right) \\
& \quad+\left[\sigma_{\alpha \alpha_{\circ}}^{(2)}\left(\theta_{\circ}, \phi_{s}-\phi_{\circ}, \theta_{s}, u_{10}\right) \cos \left(2 \Phi_{s i}\right)\right] \cos \left(2 \varphi_{w}\right) \\
& \quad+\left[\sigma_{\alpha \alpha_{\circ}}^{(2)}\left(\theta_{\circ}, \phi_{s}-\phi_{\circ}, \theta_{s}, u_{10}\right) \sin \left(2 \Phi_{s i}\right)\right] \sin \left(2 \varphi_{w}\right) .
\end{aligned}
$$

In this form, $\Phi_{s i}$ apparently depends on the absolute radiometer azimuth angle $\phi_{s}$, but this dependence is particularly simple. By recalling the definition of $\Phi_{s i}$, i.e.,

$$
\Phi_{s i}\left(\theta_{\circ}, \phi_{\circ}, \phi_{s}, \theta_{s}\right)=\tan ^{-1}\left(\frac{\sin \theta_{s} \sin \phi_{s}+\sin \theta_{\circ} \sin \phi_{\circ}}{\sin \theta_{s} \cos \phi_{s}+\sin \theta_{\circ} \cos \phi_{\circ}}\right)
$$

and by considering a change in $\phi_{s}$ and $\phi_{\circ}$ by the same angle $-\Delta \phi_{s}$, it may be shown that the shifted $\Phi_{s i}$ corresponding to the shifted $\phi_{s}$ and $\phi_{\circ}$ is related to $\Phi_{s i}$ evaluated at the original angles $\left(\theta_{\circ}, \phi_{\circ}, \phi_{s}, \theta_{s}\right)$ by

$\Phi_{s i}\left(\theta_{\circ}, \phi_{\circ}, \phi_{s}, \theta_{s}\right)=\Phi_{s i}\left(\theta_{\circ}, \phi_{\circ}-\Delta \phi_{s}, \phi_{s}-\Delta \phi_{s}, \theta_{s}\right)+\Delta \phi_{s}$.

Choosing $\Delta \phi_{s}=\phi_{s}$, we obtain the identity

$$
\begin{aligned}
\Phi_{s i}\left(\theta_{\circ}, \phi_{\circ}, \phi_{s}, \theta_{s}\right) & =\Phi_{s i}\left(\theta_{\circ}, \phi_{\circ}-\phi_{s}, 0, \theta_{s}\right)+\phi_{s} \\
& =\Phi_{s i}^{0}\left(\theta_{\circ}, \phi_{\circ}-\phi_{s}, \theta_{s}\right)+\phi_{s} .
\end{aligned}
$$

The combined cross sections may then be rewritten as

$$
\begin{aligned}
\sigma_{p}^{\prime} & \left(\theta_{\circ}, \phi_{s}-\phi_{\circ}, \theta_{s}, u_{10}, \varphi_{s}^{\prime}\right) \\
= & \sigma_{p}^{(0)}\left(\theta_{\circ}, \phi_{s}-\phi_{\circ}, \theta_{s}, u_{10}\right) \\
& +\left[\sigma_{p}^{(2)}\left(\theta_{\circ}, \phi_{s}-\phi_{\circ}, \theta_{s}, u_{10}\right) \cos \left(2 \Phi_{s i}^{0}\left(\theta_{\circ}, \phi_{\circ}-\phi_{s}, \theta_{s}\right)\right)\right] \\
& \times \cos \left(2 \varphi_{w}^{\prime}\right) \\
& +\left[\sigma_{p}^{(2)}\left(\theta_{\circ}, \phi_{s}-\phi_{\circ}, \theta_{s}, u_{10}\right) \sin \left(2 \Phi_{s i}^{0}\left(\theta_{\circ}, \phi_{\circ}-\phi_{s}, \theta_{s}\right)\right)\right] \\
& \times \sin \left(2 \varphi_{w}^{\prime}\right)
\end{aligned}
$$

where $\varphi_{w}^{\prime}=\phi_{w}-\phi_{s}$ is the downwind direction (toward which the wind is blowing) relative to the scattering (radiometer) azimuth. By introducing $\Phi_{s i}^{0}$ and the wind direction relative to the scattering azimuth $\varphi_{w}^{\prime}$, we have shifted all of the dependence on absolute radiometer azimuth and wind direction into the $\cos \left(2 \varphi_{w}^{\prime}\right)$ and $\sin \left(2 \varphi_{w}^{\prime}\right)$ factors, so the coefficients of these factors involve integrals whose integrands only depend on relative azimuth $\phi_{s}-\phi_{0}$. One may then apply the change 
of variables involving $\psi_{u h}$ discussed in Appendix A and then integrate the product of the scattering cross sections and the celestial downwelling radiation brightness temperatures over the upper hemisphere to obtain the final expression for the scattered celestial noise given by (23).

\section{ACKNOWLEDGMENT}

The authors would like to thank the two anonymous reviewers for their suggested improvements to this paper.

\section{REFERENCES}

[1] C. T. Swift and R. E. McIntosh, "Considerations for microwave remote sensing of ocean-surface salinity," IEEE Trans. Geosci. Remote Sens., vol. GRS-21, no. 4, pp. 480-491, Oct. 1983.

[2] S. H. Yueh, R. West, W. J. Wilson, F. K. Li, E. G. Njoku, and Y. Rahmat-Samii, "Error sources and feasibility for microwave remote sensing of ocean surface salinity," IEEE Trans. Geosci. Remote Sens., vol. 39, no. 5, pp. 1049-1060, May 2001.

[3] D. M. Le Vine and S. Abraham, "Galactic noise and passive microwave remote sensing from space at L-band," IEEE Trans. Geosci. Remote Sens., vol. 42, no. 1, pp. 119-129, Jan. 2004.

[4] D. M. Le Vine, S. Abraham, Y. Kerr, W. Wilson, N. Skou, and S. Søbjærg, "Comparison of model prediction with measurements of galactic background noise at L-band," IEEE Trans. Geosci. Remote Sens., vol. 43, no. 9, pp. 2018-2023, Sep. 2005.

[5] W. Reich, "A radio continuum survey of the northern sky at 1420 MHz-Part I," Astron. Astrophys., Suppl. Ser., vol. 48, pp. 219-297, Jul. 1982.

[6] P. Reich and W. Reich, "A radio continuum survey of the northern sky at $1420 \mathrm{MHz}$-Part II," Astron. Astrophys., Suppl. Ser., vol. 63, no. 2, pp. 205-292, Feb. 1986

[7] P. Reich, J. C. Testori, and W. Reich, "A radio continuum survey of the northern sky at $1420 \mathrm{MHz}$ - The atlas of contour maps," Astron. Astrophys., vol. 376, pp. 861-877, 2001.

[8] E. M. Arnal, E. Bajaja, J. J. Larrarte, R. Morras, and W. G. L. Pöppel, “A high sensitivity HI survey of the sky at $\delta \leq-25^{\circ}$," Astron. Astrophys., Suppl. Ser, vol. 142, no. 1, pp. 35-40, Feb. 2000.

[9] J. C. Testori, P. Reich, J. A. Bava, F. R. Colomb, E. E. Hurrel, J. J. Larrarte, W. Reich, and A. J. Sanz, "A radio continuum survey of the southern sky at $1420 \mathrm{MHz}$-Observations and data reduction," Astron. Astrophys., vol. 368, no. 3, pp. 1123-1132, Mar. 2001.

[10] D. Hartmann and W. Butler Burton, Atlas of Galactic Neutral Hydrogen. Cambridge, U.K.: Cambridge Univ. Press, 1997.

[11] S. Schmidl Søbjærg, J. Rotbøll, and N. Skou, "Wind effects and angular dependence at L-band polarimetric data: First results of LOSAC," presented at the Proc. 1st Results Workshop: EuroSTARRS, WISE, LOSAC Campaigns, pp. 181-189, P. Fletcher Ed., Mar. 2003, Paper ESA SP-525.

[12] J. Etcheto, E. P. Dinnat, J. Boutin, A. Camps, J. Miller, S. Contardo, J. Wesson, J. Font, and D. Long, "Wind speed effect on L-band brightness temperature inferred from EuroSTARRS and WISE 2001 field experiments," IEEE Trans. Geosci. Remote Sens., vol. 42, no. 10, pp. 22062213, Oct. 2004.

[13] A. Camps, J. Font, M. Vall-llossera, C. Gabarró, I. Corbella, N. Duffo, F. Torrres, S. Blanch, A. Aguasca, R. Villarino, L. Enrique, J. J. Miranda, J. J. Arenas, A. Julià, J. Etcheto, V. Caselles, A. Weill, J. Boutin, S. Contardo, R. Niclós, R. Rivas, S. C. Reising, P. Wursteisen, M. Berger, and M. Martín-Neira, "The WISE 2000 and 2001 field experiments in support of the SMOS mission: Sea surface L-band brightness temperature observations and their application to sea surface salinity retrieval," IEEE Trans. Geosci. Remote Sens., vol. 42, no. 4, pp. 804-823, Apr. 2004.

[14] W. J. Wilson, S. H. Yueh, S. J. Dinardo, S. L. Chazanoff, A. Kitiyakara, F. K. Li, and Y. Rahmat-Samii, "Passive active L- and S-band (PALS) microwave sensor for ocean salinity and soil moisture measurements," IEEE Trans. Geosci. Remote Sens., vol. 39, no. 5, pp. 1039-1048, May 2001.

[15] P. Waldteufel, "Impact of the variable angular apodization function on galactic contribution," CBSA, Toulouse, France, Tech. Rep. SO-TNCBSA-GS-0013, Apr. 2006.

[16] J. P. Hollinger, "Passive microwave measurements of sea surface roughness," IEEE Trans. Geosci. Electron., vol. GE-9, no. 3, pp. 165-169, Jul. 1971.
[17] C. T. Swift, "Microwave radiometer measurements of the Cape Cod canal," Radio Sci., vol. 9, no. 7, pp. 641-653, 1974.

[18] W. J. Webster, T. T. Wilheit, D. B. Ross, and P. Gloersen, "Spectral characteristics of the microwave emission from a wind-driven foam-covered sea," J. Geophys. Res., vol. 81, no. 18, pp. 3095-3099, 1976.

[19] N. Reul, J. E. Tenerelli, B. Chapron, and D. Vandemark, "Reanalysis of Skylab S-194 L-band data in view of validating sea surface roughness corrections for salinity measurements from space," in Proc. IGARSS, 2005, pp. 2572-2575.

[20] A. Camps, J. Font, J. Etcheto, V. Caselles, A. Weill, I. Corbella, M. Vall-llossera, N. Duffo, F. Torres, R. Villarino, L. Enrique, A. Julià, C. Gabarró, J. Boutin, E. Rubio, S. C. Reising, P. Wursteisen, M. Berger, and M. Martín-Neira, "Sea surface emissivity observations at L-band: First results of the Wind and Salinity Experiment WISE 2000," IEEE Trans. Geosci. Remote Sens., vol. 40, no. 10, pp. 2117-2130, Oct. 2002.

[21] A. Guissard, "Mueller and Kennaugh matrices in radar polarimetry," IEEE Trans. Geosci. Remote Sens., vol. 32, no. 3, pp. 590-597, May 1994.

[22] J. P. Claassen and A. K. Fung, "The recovery of polarized apparent temperature distributions of flat scenes from antenna temperature measurements," IEEE Trans. Antennas Propag., vol. AP-22, no. 3, pp. $433-$ 442, May 1974.

[23] S. H. Yueh, "Estimates of Faraday rotation with passive microwave polarimetry for microwave remote sensing of earth surfaces," IEEE Trans. Geosci. Remote Sens., vol. 38, no. 5, pp. 2434-2438, Sep. 2000.

[24] N. Skou and D. Hoffman-Bang, "L-band radiometers measuring salinity from space: Atmospheric propagation effects," IEEE Trans. Geosci. Remote Sens., vol. 43, no. 10, pp. 2210-2217, Oct. 2005. [Online]. Available: http://server.oersted.dtu.dk/publications/p.php?1822

[25] T. M. Elfouhaily and C.-A. Guérin, "A critical survey of approximate scattering wave theories from random rough surfaces," Waves Random Media, vol. 14, no. 4, pp. R1-R40, Oct. 2004.

[26] A. C. Ludwig, "The definition of cross polarization," IEEE Trans. Antennas Propag., vol. AP-21, no. 1, pp. 116-119, Jan. 1973.

[27] P. Waldteufel and G. Caudal, "About off-axis radiometric polarimetric measurements," IEEE Trans. Geosci. Remote Sens., vol. 40, no. 6 , pp. 1435-1439, Jun. 2002.

[28] N. Reul, J. Tenerelli, B. Chapron, and P. Waldteufel, "Modeling sun glitter at L-band for sea surface salinity remote sensing with SMOS," IEEE Trans. Geosci. Remote Sens., vol. 45, no. 7, pp. 2073-2087, Jul. 2007.

[29] P. Beckmann and A. Spizzichino, The Scattering of Electromagnetic Waves From Rough Surfaces. New York: Macmillan, 1963.

[30] A. G. Voronovich, "Small-slope approximation in wave scattering by rough surfaces," Sov. Phys._JETP, vol. 62, no. 1, pp. 65-70, 1985.

[31] A. G. Voronovich and V. U. Zavorotny, "Theoretical model for scattering of radar signals in Ku and C-bands from a rough sea surface with breaking waves," Waves Random Media, vol. 11, no. 3, pp. 247-269, Jul. 2001.

[32] T. Elfouhaily, S. Guignard, R. Awadallahand, and D. R. Thompson, "Local and non-local curvature approximation: A new asymptotic theory for wave scattering," Waves Random Media, vol. 13, no. 4, pp. 321-337, Oct. 2003.

[33] L. A. Klein and C. T. Swift, "An improved model for the dielectric constant of sea water at microwave frequencies," IEEE Trans. Antennas Propag., vol. AP-25, no. 1, pp. 104-111, Jan. 1977.

[34] V. N. Kudryavtsev, V. K. Makin, and B. Chapron, "Coupled sea surfaceatmosphere model 2. Spectrum of short wind waves," J. Geophys. Res., vol. 104, no. C4, pp. 7625-7639, 1999.

[35] C. Cox and W. Munk, Slopes of the Sea Surface Deduced From Photographs of Sun Glitter. Berkeley, CA: Univ. California Press Berkeley, 1956, pp. 401-488.

[36] V. Kudryavtsev, D. Hauser, G. Caudal, and B. Chapron, "A semi-empirical model of the normalized radar cross-section of the sea surface-Part 1. Background model," J. Geophys. Res., vol. 108, no. C3, 8054, 2003. DOI:10.1029/2001JC001003.

[37] A. Mouche, D. Hauser, and V. Kudryavtsev, "Radar scattering of the ocean surface and sea-roughness properties: A combined analysis from dual-polarizations airborne radar observations and models in C-band," J. Geophys. Res., vol. 111, no. C9, C09 004, 2006. DOI:10.1029/ 2005JC003166.

[38] S. Blanch and A. Aguasca, "Seawater dielectric permittivity model from measurements at L-band," in Proc. IGARSS, 2004, pp. 1362-1365.

[39] A. Camps, J. Font, M. Vall-llossera, R. Villarino, C. Gabarró, L. Enrique, J. Miranda, I. Corbella, N. Duffo, F. Torres, S. Blanch, A. Aguasca, and R. Sabia, "From the determination of sea emissivity to the retrieval of salinity: Recent contributions to the SMOS mission from the UPC and ICM,” in Proc. IGARSS, 2006, pp. 1697-1701. 
[40] W. Reich, P. Reich, and E. Fürst, "The Effelsberg $21 \mathrm{~cm}$ radio continuum survey of the galactic plane between $L=357^{\circ}$ and $L=95.5^{\circ}$," Astron. Astrophys., Suppl. Ser., vol. 83, no. 3, pp. 539-568, Jun. 1990.

[41] W. Reich, P. Reich, and E. Fuerst, " $21 \mathrm{~cm}$ radio continuum surveyPart I," VizieR Online Data Catalog, vol. 408, p. 30 539, Nov. 1997.

[42] J. T. Johnson and M. Zhang, "Theoretical study of the small slope approximation for ocean polarimetric thermal emission," IEEE Trans. Geosci. Remote Sens., vol. 37, no. 5, pp. 2305-2316, Sep. 1999.

[43] V. G. Irisov, "Small-slope expansion for thermal and reflected radiation from a rough surface," Waves Random Media, vol. 7, no. 1, pp. 1-10, Jan. 1997.

[44] J. Font, J. Boutin, N. Reul, P. Waldteufel, C. Gabarró, S. Zine, and J. Tenerelli, "SMOS sea surface salinity level 2 algorithm theoretical baseline document-Issue 2," European Space Agency ESTEC Contract 18933/05/nl/ff, 2007.

[45] S. H. Yueh, R. Kwok, and S. V. Nghiem, "Polarimetric scattering and emission properties of targets with reflection symmetry," Radio Sci., vol. 29, no. 6, pp. 1409-1420, Nov./Dec. 1994.

[46] A. J. Camps and S. C. Reising, "Wind direction azimuthal signature in the Stokes emission vector from the ocean surface at microwave frequencies," Microw. Opt. Technol. Lett., vol. 29, no. 6, pp. 426-432, Jun. 2001.

[47] J. T. Johnson, "An efficient two-scale model for the computation of thermal emission and atmospheric reflection from the sea surface," IEEE Trans. Geosci. Remote Sens., vol. 44, no. 3, pp. 560-568, Mar. 2006.

[48] S. H. Yueh, R. Kwok, F. K. Li, S. V. Nghiem, and W. J. Wilson, "Polarimetric passive remote sensing of ocean wind vectors," Radio Sci., vol. 29, no. 4, pp. 799-814, Jul./Aug. 1994.

[49] M. Wolleben, T. L. Landecker, W. Reich, and R. Wielebinski, "An absolutely calibrated survey of polarized emission from the northern sky at $1.4 \mathrm{GHz}$-Observations and data reduction," Astron. Astrophys., vol. 448, no. 1, pp. 411-424, Mar. 2006.

[50] O. Germain, G. Ruffini, F. Soulat, M. Caparrini, B. Chapron, and P. Silvestrin, "The Eddy experiment: GNSS-R speculometry for directional sea-roughness retrieval from low altitude aircraft," Geophys. Res. Lett., vol. 31, L21307, 2004. DOI:10.1029/2004GL020991.

[51] M. Sánchez-Nogales, F. Pirondini, and J. A. G. Abeytua, Earth Explorer Mission CFI Software: Mission Conventions Document. Madrid, Spain: DEIMOS Space S.L., Jul. 2003. Technical note.

[52] N. Reul, J. Tenerelli, N. Floury, and B. Chapron, "Earth-viewing L-band radiometer sensing of sea surface scattered celestial sky radiationPart II: Application to SMOS," IEEE Trans. Geosci. Remote Sens., vol. 46, no. 3, Mar. 2008, to be published



Joseph E. Tenerelli received the B.S. degree in atmospheric sciences from the University of Washington, Seattle, in 1994.

From 1999 to 2005, he was a Research Associate with the Rosenstiel School of Marine and Atmospheric Science, University of Miami, Miami, FL, where he was part of a team that developed a coupled atmosphere-ocean-surface wave model with vortexfollowing mesh refinement suitable for simulating hurricanes. Since April 2005, he has been a Research Engineer with the Laboratoire d'Océanographie Spatiale, Institut Français de Recherche et d'Exploitation de la Mer, Plouzané, France, as part of a team that is developing an algorithm for retrieving sea surface salinity from L-band radiometric measurements (the European Space Agency's Soil Moisture and Ocean Salinity project).

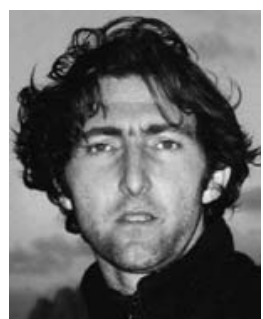

Nicolas Reul received the B.S. degree in marine science engineering from Toulon University, Toulon, France, in 1993 and the Ph.D. degree in physics (fluid mechanics) from the University of Aix-Marseille II, Marseille, France, in 1998.

From 1999 to 2001, he was with the Department of Applied Marine Physics, Rosenstiel School of Marine and Atmospheric Science, University of Miami, Miami, FL, as a Postdoctoral Researcher in the team of Prof. M. Donelan. Since 2001, he has been a Research Scientist with the Spatial Oceanography Group, Laboratoire d'Océanographie Spatiale, Institut Français de Recherche et d'Exploitation de la Mer, Plouzané, France, where is responsible for activities concerning the SMOS satellite mission. The focus of his research program is the improvement of the understanding of the physical processes in the air-sea interface and passive/active remote sensing of the ocean surface. He has experience in applied mathematics, physical oceanography, and electromagnetic wave theory and its application to ocean remote sensing. He is a member of the European Space Agency/SMOS Science Advisory Group.



Alexis A. Mouche received the D.E.A. in physics for remote sensing from the University of Pierre et Marie Curie, Paris, France, in 2002 and the Ph.D. degree in physics, focusing on remote sensing, from the University of Versailles Saint-Quentin-en-Yvelines, Versailles, France, in 2005.

From 2002 to 2005 , he was a Ph.D. student at the Centre d'Étude des Environnements Terrestres et Planétaires/Institut Pierre Simon Laplace-Centre National de la Recherche Scientifique, Saint Maur, France. Since January 2006, he has been a Postdoctoral Scientist with the Centre National d'Études Spatiales, Paris. He is also with the Spatial Oceanography Group, Laboratoire d'Océanographie Spatiale, Institut Français de Recherche et d'Exploitation de la Mer, Plouzané, France. His work is dedicated mainly to the study of the ocean surface by active/passive microwave sensors.

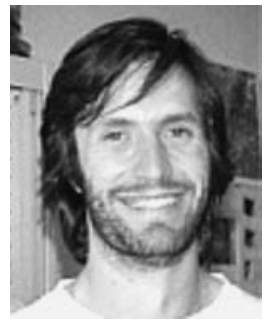

Bertrand Chapron received the B.Eng. degree from the Institut National Polytechnique de Grenoble, Grenoble, France, in 1984 and the Ph.D. degree in physics (fluid mechanics) from the University of Aix-Marseille II, Marseille, France, in 1988.

He was with the NASA Goddard Space Flight Center Wallops Flight Facility, Wallops Island, VA, for three years as a Postdoctoral Research Associate. He is currently a Research Scientist and the Head of the Spatial Oceanography Group, Laboratoire d'Océanographie Spatiale, Institut Français de Recherche et d'Exploitation de la Mer, Plouzané, France, where he is responsible for the Centre ERS Archivage et Traitement. He has been a Coinvestigator and Principal Investigator in several projects of the European Space Agency (e.g., ENVISAT and Global Navigation Satellite System), NASA, and Centre National d'Études Spatiales (e.g., TOPEX/POSEIDON and JASON). He was also responsible (with H. Johnsen of NORUT) for the ENVISAT ASAR-wave mode algorithms and scientific preparation for the ENVISAT wind and wave products. He has experience in applied mathematics, physical oceanography, and electromagnetic wave theory and its application to ocean remote sensing. 